\title{
The Development of Tyrosyl-DNA Phosphodiesterase 1 Inhibitors. Combination of Monoterpene and Adamantine Moieties via Amide or Thioamide Bridges
}

\author{
Arina A. Chepanova ${ }^{1}$, Evgenii S. Mozhaitsev ${ }^{2}$, Aldar A. Munkuev ${ }^{2,3}$, Evgeniy V. Suslov ${ }^{2}$, \\ Dina V. Korchagina ${ }^{2}$, Olga D. Zakharova ${ }^{1}$, Alexandra L. Zakharenko ${ }^{1}$, Jinal Patel ${ }^{4}$, \\ Daniel M. Ayine-Tora ${ }^{4}$, Jóhannes Reynisson ${ }^{5, * \mathbb{C}}$, Ivanhoe K. H. Leung ${ }^{4,6}{ }^{\mathbb{D}}$, \\ Konstantin P. Volcho ${ }^{2,3}$ (-) Nariman F. Salakhutdinov ${ }^{2,3}$ and Olga I. Lavrik ${ }^{1,3}$ \\ 1 Institute of Chemical Biology and Fundamental Medicine, Siberian Branch of Russian Academy of Sciences, \\ 8, Lavrentiev Ave., Novosibirsk 630090, Russia \\ 2 N.N. Vorozhtsov Novosibirsk Institute of Organic Chemistry, Siberian Branch of Russian Academy of \\ Sciences, 9, Lavrentiev Ave., Novosibirsk 630090, Russia \\ 3 Novosibirsk State University, 2, Pirogova Str., Novosibirsk 630090, Russia \\ 4 School of Chemical Sciences, The University of Auckland, Private Bag 92019, Victoria Street West, \\ Auckland 1142, New Zealand \\ 5 School of Pharmacy, Keele University, Hornbeam Building, Staffordshire ST5 5BG, UK \\ 6 Maurice Wilkins Centre for Molecular Biodiscovery, The University of Auckland, Private Bag 92019, \\ Victoria Street West, Auckland 1142, New Zealand \\ * Correspondence: j.reynisson@keele.ac.uk; Tel.: +44-(0)-178-2733-985
}

Received: 12 June 2019; Accepted: 3 July 2019; Published: 9 July 2019

Featured Application: Inhibition of Tdp1 has the potential to increase the potency of the anticancer drugs topotecan and irinotecan, topoisomerase 1 poisons. Furthermore, Tdp1 inhibitors can facilitate the therapeutic use of these drugs in other cancer types.

\begin{abstract}
Eleven amide and thioamide derivatives with monoterpene and adamantine substituents were synthesised. They were tested for their activity against the tyrosyl-DNA phosphodiesterase 1 DNA (Tdp1) repair enzyme with the most potent compound $47 \mathrm{a}$, having an $\mathrm{IC}_{50}$ value of 0.64 $\mu \mathrm{M}$. When tested in the A-549 lung adenocarcinoma cell line, no or very limited cytotoxic effect was observed for the ligands. However, in conjunction with topotecan, a well-established Topoisomerase 1 (Top1) poison in clinical use against cancer, derivative 46a was very cytotoxic at $5 \mu \mathrm{M}$ concentration, displaying strong synergism. This effect was only seen for $46 \mathrm{a}\left(\mathrm{IC}_{50}-3.3 \mu \mathrm{M}\right)$ albeit some other ligands had better $\mathrm{IC}_{50}$ values. Molecular modelling into the catalytic site of Tdp1 predicted plausible binding mode of $46 a$, effectively blocking access to the catalytic site.
\end{abstract}

Keywords: topotecan; A-549 lung adenocarcinoma cell line 1; Topoisomerase 1; molecular modelling; chemical space; synergy; thermal shift assay; intrinsic tryptophan fluorescence binding assay; fluorescence biosensor assay

\section{Introduction}

Camptothecin derivatives (CPTs) such as topotecan, irinotecan and belotecan are common chemotherapy agents that are used for the treatment of cancers. These therapeutic agents are used either as monotherapy or in combination with other anticancer drugs for the treatments of small cell lung cancer, metastatic colon and rectal carcinoma [1,2]. Belotecan was recently approved in South Korea for non-small-cell lung cancer and ovarian cancer [2]. 
The target of CPTs is topoisomerase I (TOP1) [3]. TOP1 plays an essential role during transcription, replication and repair of DNA by resolving helical duplex DNA topological problems. First, the enzyme forms a complex with double-strand DNA, a tyrosine residue at the TOP1 active site then introduces a single-strand break by catalysing the formation of a 3'-tyrosyl-phosphodiester bond, leading to the covalent linking of TOP1 and the 3'-phosphoryl end of the DNA. After rotation of the 5 -DNA end around the non-cleaved strand, the integrity of the DNA is restored, which is then followed by enzyme dissociation [4]. Binding of CPTs with the transient TOP1-DNA complex inhibits TOP1 dissociation, which subsequently leads to the formation of irreversible covalently-bound TOP1-DNA complexes, thus blocking replication and transcription [5]. The accumulation of DNA lesions halts cellular processes and results in cell death.

Repair enzymes such as tyrosyl-DNA phosphodiesterase (Tdp1), 3'-flap endonucleases, and base excision repair (BER) complexes cleave covalent TOP1-DNA complexes, thus initiating DNA integrity recovery. The Tdp1 enzyme hydrolyses the 3'-phosphotyrosyl bond between single-strand DNA and TOP1, which is formed as a result of TOP1 hydrolysis catalysed by proteases. In addition, Tdp1 is involved in the elimination of other lesions at the $3^{\prime}$-end of DNA, for example, $3^{\prime}$-phosphoglycolate, and repair of apurinic/apyrimidinic sites generated by oxidative and free radical DNA damage [6-8]. Therefore, in the context of the molecular mechanism of CPTs, Tdp1 catalyses the dissociation of the TOP1-DNA complexes that are trapped by CPTs and; therefore, eliminating the therapeutic effect of TOP1 poisons. Moreover, Tdp1 activity was found to cause drug resistance of some cancer cell lines [9-11] and Tdp1 knockout cells were demonstrated to show increased sensitivity to CPT [12] and irinotecan [10]. Thus, enhancing anticancer the therapeutic effect can be achieved with the simultaneous administration of CPTs and Tdp1 inhibitors [13]. Furthermore, Tdp1 inhibition can lead to synergistic effect with anticancer agents with other mechanisms of action, including bleomycin and its derivatives as well as monofunctional alkylating agents or even radiotherapy [14,15].

A wealth of Tdp1 inhibitors have been identified so far with various molecular scaffolds (see Figure 1) and potency down to nano-molar activity [16-40].

It is known that monoterpene derivatives have various biological activities [41]. Interestingly, potent Tdp1 inhibitory activity was obtained for citral, citronellal and perillaldehyde derivatives of usnic acid, citronellal derivative 17 with $\mathrm{IC}_{50}=0.43 \mu \mathrm{M}$ ) [34] and 7-hydroxycoumarin, most potent being 18 with $\mathrm{IC}_{50}=0.33 \mu \mathrm{M}$, the structures are shown in Figure 2. Furthermore, derivative $\mathbf{1 8}$ had synergistic effect with CPT, 18 increasing CPT cytotoxicity eight-fold in the breast adenocarcinoma MCF-7 cell line [42]. Monoterpene derivatives of substituted anilines were found to be active at low micromolar concentrations $\left(\mathrm{IC}_{50}=1.29 \mu \mathrm{M}\right)$ for 19 and for $20\left(\mathrm{IC}_{50}=0.79 \mu \mathrm{M}\right)$ (Figure 2) [43]. Additionally, potent inhibitory activity was found for derivatives containing adamantane. Bile acid amide 21 inhibited Tdp1 with $\mathrm{IC}_{50}=0.47 \mu \mathrm{M}$ [44], whilst octahydro- $2 H$-chromen-4-ol derivative $22 \mathrm{IC}_{50}$ was $1.24 \mu \mathrm{M}$ (see Figure 2) [45]. It was also demonstrated that compounds combining adamantane/heteroadamantane and monoterpene fragments are active against Tdp1 [46,47]. Moderate activity at micromolar concentrations $\left(\mathrm{IC}_{50} \sim 15 \mu \mathrm{M}\right)$ was demonstrated for diazaadamantanes containing citronellal and citral moieties $\mathbf{2 3}$ and $\mathbf{2 4}$ (Figure 2) [46]. The adamantane derivatives $\mathbf{2 5}$ and $\mathbf{2 6}$ (Figure 2) had activity at $\mathrm{IC}_{50}=6.0$ and $3.5 \mu \mathrm{M}$, respectively [47]. A synergistic effect was found for the simultaneous action of $\mathbf{2 5}$ or $\mathbf{2 6}$ and topotecan. Derivative $\mathbf{2 6}$ increased cytotoxic effect of topotecan five-fold whilst 25 increased cytotoxicity two-fold in the colon carcinoma HCT-116 cell line [47]. Monoterpene derivatives of 1- and 2-aminoadamantane 27 and 28 decreased Tdp 1 activity by $50 \%$ at 6.6 and $5.5 \mu \mathrm{M}$, respectively [48]. 


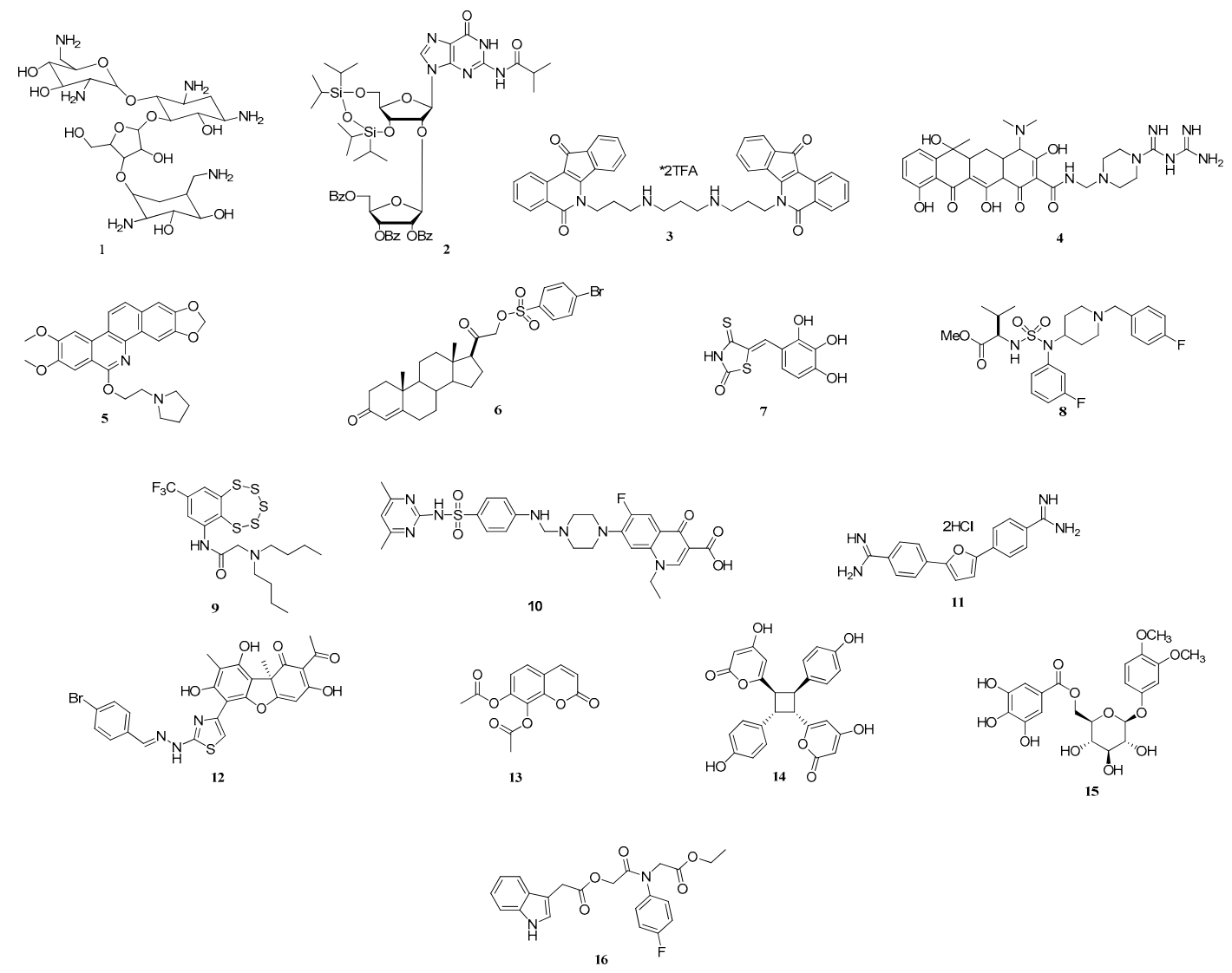

Figure 1. The chemical structures of known Tdp1 inhibitors, a remarkable diversity is accommodated by the enzyme.
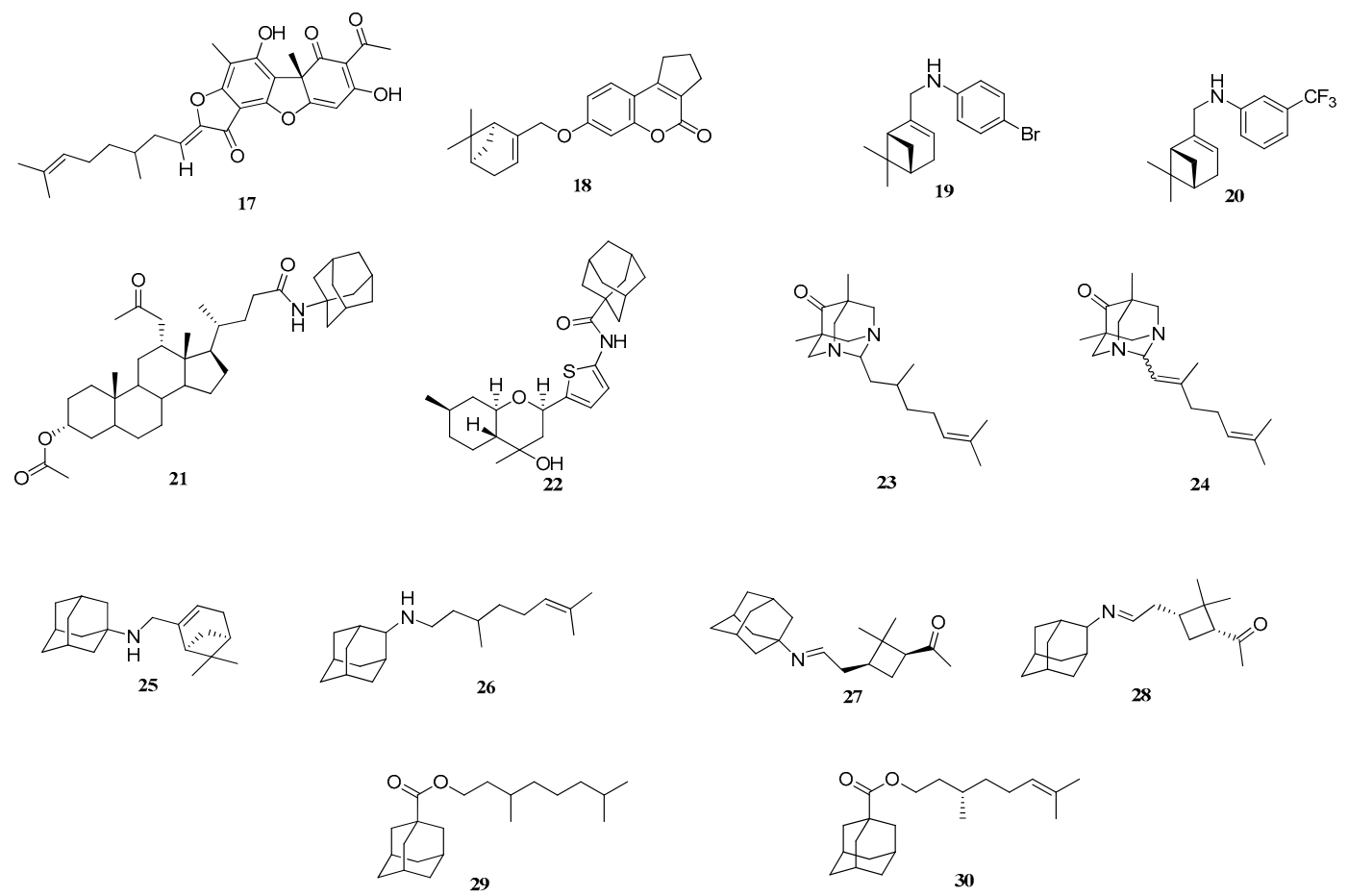

Figure 2. Monoterpenes and adamantane/diazaadmanatane derivatives with Tdp1 inhibitory activity.

Previously we synthesized a number of esters of 1-adamantane carboxylic acid containing fragments of acyclic, monocyclic and bicyclic monoterpenoids. A potent Tdp1 inhibitory activity 
with $\mathrm{IC}_{50}$ ranging from 0.9 to $4 \mu \mathrm{M}$ was achieved [49]. It was found that acyclic derivatives 29 $\left(\mathrm{IC}_{50}=0.89 \mu \mathrm{M}\right)$ and $30\left(\mathrm{IC}_{50}=0.92 \mu \mathrm{M}\right)$ are in Figure 2$)$ had more potent activity than monocyclic or bicyclic derivatives. Additionally, ester 29 increased topotecan cytotoxicity [49]. Drugs containing the ester moiety often have metabolic instability due to hydrolysis by carboxylesterases [50]. To mitigate this problem, amides can be used. Therefore, we investigated the substitution of esters by amides and thioamides in combining adamantane and acyclic monoterpene fragments on Tdp1 inhibitory activity.

\section{Materials and Methods}

\subsection{Chemistry}

Adamantane 1-carbonyl chloride (97\%), nerol (97\%) and trimethylsulfoxonium iodide (98\%) were purchased from Acros organics; 3,7-dymethiloctanol ( $\geq 98 \%$ ) and boron trifluoride diethyl etherate were purchased from Sigma-Aldrich; citronellic acid (94\%) was purchased from Alfa Aesar; adamantan-2-one $(\geq 99 \%)$ was purchased from Merck. Toluene was freshly distilled under sodium before use. Column chromatography was performed with silica gel (60-230 $\mu$, Macherey-Nagel), solution containing from $0 \%$ to $5 \%$ ethyl acetate in hexane. ${ }^{1} \mathrm{H}$ - and ${ }^{13} \mathrm{C}$-NMR spectra were registered on a Bruker Avance- $\mathrm{III}$ 600 spectrometer $\left(600.30 \mathrm{MHz}\left({ }^{1} \mathrm{H}\right)\right.$ and $150.95 \mathrm{MHz}\left({ }^{13} \mathrm{C}\right)$ in $\left.\mathrm{CDCl}_{3}\right)$. Chemical shifts obtained are given in ppm, relative to residual chloroform $\left(\delta_{\mathrm{H}} 7.24 \mathrm{ppm}, \delta_{\mathrm{C}} 76.90 \mathrm{ppm}\right)$, and J are given in $\mathrm{Hz}$. The structures of the products were determined by analyzing their ${ }^{1} \mathrm{H}$ NMR spectra, J-modulated ${ }^{13} \mathrm{C}$ NMR spectra (JMOD), ${ }^{13} \mathrm{C}-{ }^{1} \mathrm{H}$-type 2D heteronuclear correlation with one bond (HSQC, ${ }^{1} \mathrm{~J} 145$ $\mathrm{Hz}$ ) and long-range spin-spin coupling constants $\left(\mathrm{HMBC},{ }^{2,3} \mathrm{~J} 7 \mathrm{~Hz}\right.$ ) and ${ }^{1} \mathrm{H}-{ }^{1} \mathrm{H}$ double-resonance spectra (COSY, NOESY). Numeration of atoms in the compounds (see Supplementary Materials, Figures S7-S15) is given for assigning the signals in the NMR spectra and does not coincide with the nomenclature of the compounds. The elemental composition of compounds was determined from high-resolution mass spectra (HR-MS) recorded on a DFS Thermo Scientific spectrometer in full scan mode $(0-500 \mathrm{~m} / \mathrm{z}, 70 \mathrm{eV}$ electron impact ionization, direct sample injection). The conversion of reagents, the content of the compounds in fractions during chromatography and the purity of the target compounds was determined using gas chromatography methods: 7820A gas chromatograph (Agilent Tech., USA), flame-ionization detector, HP-5 capillary column $(0.25 \mathrm{~mm} \times 3 \mathrm{~m} \times 0.25 \mu \mathrm{m})$, helium carrier gas (flow rate $2 \mathrm{~mL} / \mathrm{min}$, flow division 99:1), temperature range from 120 to $280{ }^{\circ} \mathrm{C}$, heating $20^{\circ} \mathrm{C} / \mathrm{min}$. The purity of the target compounds for biological testing was confirmed to be more than $95 \%$.

\subsubsection{Synthesis of Amines 36, 37}

Bromides 40, 41 were synthesized from 3,7-dimethyloctanol 38 and nerol 39 ( $1.0 \mathrm{~g}, 6.5 \mathrm{mmol}$, $2.5 \mathrm{eq})$ by stirring of with $\mathrm{PBr}_{3}(0.24 \mathrm{~mL}, 2.6 \mathrm{mmol}, 1 \mathrm{eq})$ in diethyl ether (38) and THF (39) solutions (10 $\mathrm{mL}$ of each solvents) at cooling according to the methods [42,51]. Then, the derivatives $\mathbf{4 0 , 4 1}$ obtained were used without further purification. To solution $\mathbf{4 0}$ and $\mathbf{4 1}(1.2 \mathrm{~g}, 5.4 \mathrm{mmol}, 1 \mathrm{eq})$ in dry DMF $(40 \mathrm{~mL})$ potassium phthalimide $(1.0 \mathrm{~g}, 5.4 \mathrm{mmol}, 1 \mathrm{eq})$ was added, the reaction mixture was stirred at $50{ }^{\circ} \mathrm{C}$ until the reaction completed, treated with water $(100 \mathrm{~mL})$ and extracted by $\mathrm{CHCl}_{3}$. The extract was washed by $5 \% \mathrm{NaOH}$ solution $(50 \mathrm{~mL})$, brine $(50 \mathrm{~mL})$ and evaporated with water and toluene consequentially. Resulted residues were purified by column chromatography with yielding phthalimides $42(1.3 \mathrm{~g}, 4.5 \mathrm{mmol}$, yield $95 \%)$ and $\mathbf{4 3}(1.1 \mathrm{~g}$, $4.0 \mathrm{mmol}$, yield $74 \%)$. To solution of 42 and $43(1.1 \mathrm{~g}, 4.0 \mathrm{mmol}, 1 \mathrm{eq})$ in methanol $(30 \mathrm{~mL})$, ethylenediamine $(0.53 \mathrm{~mL}, 8.0 \mathrm{mmol}, 2 \mathrm{eq})$ was added, the mixture was refluxed until the reaction completed, treated with water $(30 \mathrm{~mL})$ and extracted by $\mathrm{Et}_{2} \mathrm{O}$. The organic layer was washed by $5 \% \mathrm{NaOH}(30 \mathrm{~mL})$, brine $(30 \mathrm{~mL})$ and dried under $\mathrm{Na}_{2} \mathrm{SO}_{4}$. The solvent was evaporated and hexane $(30 \mathrm{~mL})$ was added to the residue. The solution was filtered and the solvent was distilled off yielding $36(0.34 \mathrm{~g}, 2.4 \mathrm{mmol}$, yield 55\%) and 37 (0.57 g, $3.7 \mathrm{mmol}$, yield $94 \%$ ). 


\subsubsection{General Procedure for Amides $44 a, b ; 45 a, b ; 50 a, b$}

Citronellic acid $(0.30 \mathrm{~g}, 1.7 \mathrm{mmol}, 1 \mathrm{eq})$ was previously refluxed with thionyl chloride $(0.25 \mathrm{~mL}, 3.5$ mmol, 2 eq) in toluene ( $15 \mathrm{~mL}) 2 \mathrm{~h}$. The solvent was distilled off and the citronellic acid chloride 48 was obtained ( $0.32 \mathrm{~g}, 1.7 \mathrm{mmol}$, yield $98 \%)$. To solution of carboxylic acid chlorides $31,32(0.16 \mathrm{~g}, 0.79 \mathrm{mmol}$, $1 \mathrm{eq}), 48(0.15 \mathrm{~g}, 0.79 \mathrm{mmol}, 1 \mathrm{eq})$ in dry toluene $(20 \mathrm{~mL})$ amines 36,37 (0.12 g, $0.79 \mathrm{mmol}, 1 \mathrm{eq})$, as well as 1- and 2-aminoadamantanes $49 \mathbf{a}, \mathbf{b}(0.12 \mathrm{~g}, 0.79 \mathrm{mmol}, 1 \mathrm{eq})$ and trimethylamine $(0.11 \mathrm{~mL}, 0.79$ mmol, 1 eq) were added at $0{ }^{\circ} \mathrm{C}$. The mixtures were stirred overnight at room temperature, evaporated, dissolved in EtOAc $(30 \mathrm{~mL})$, washed by $5 \% \mathrm{NaOH}(20 \mathrm{~mL})$, brine $(20 \mathrm{~mL})$ and dried with $\mathrm{Na}_{2} \mathrm{SO}_{4}$. The solvent was distilled off under reduced pressure and the solid obtained was purified by column chromatography yielding amides $44 a, b ; 45 a, b ; 50 a, b$.

\subsubsection{N-(3,7-Dimethyloctyl)adamantane-1-carboxamide 44a}

Yield 85\%. ${ }^{1} \mathrm{H}-\mathrm{NMR}\left(\mathrm{CDCl}_{3}\right): 0.83 \mathrm{~d}(6 \mathrm{H}, \mathrm{J}(20,19)=\mathrm{J}(21,19)=6.6 \mathrm{~Hz}, 3 \mathrm{H}-20,3 \mathrm{H}-21), 0.86 \mathrm{~d}$ $(3 \mathrm{H}, \mathrm{J}(22,15)=6.6 \mathrm{~Hz}, 3 \mathrm{H}-22), 1.04-1.14 \mathrm{~m}(3 \mathrm{H}, \mathrm{H}-16,2 \mathrm{H}-18), 1.17-1.30 \mathrm{~m}\left(4 \mathrm{H}, \mathrm{H}^{\prime}-14, \mathrm{H}^{\prime}-16,2 \mathrm{H}-17\right)$, 1.37-1.52 m (3H, H'-14, H-15, H-19), 1.63-1.73 m (6H, 2H-4, 2H-6, 2H-10), $1.81 \mathrm{~d}\left(6 \mathrm{H},{ }^{3} \mathrm{~J}=3.0 \mathrm{~Hz}, 2 \mathrm{H}-2\right.$, 2H-8, 2H-9), 1.98-2.03 m (3H, H-3, H-5, H-7), 3.15-3.28 m (2H, 2H-13), 5.50 br. s (1H, NH). ${ }^{13} \mathrm{C}-\mathrm{NMR}$ $\left(\mathrm{CDCl}_{3}\right): 40.39$ (s, C-1), 39.18 (t, C-2, C-8, C-9), 28.03 (d, C-3, C-5, C-7), 36.42 (t, C-4, C-6, C-10), 177.66 (s, C-11), 37.33 (t, C-13), 36.59 (t, C-14), 30.59 (d, C-15), 36.99 (t, C-16), 24.52 (t, C-17), 39.07 (t, C-18), 27.81 (d, C-19), 22.47, 22.56 (2q, C-20, C-21), 19.46 (q, C-22). HR MS: $319.2871\left([M]^{+}, \mathrm{C}_{21} \mathrm{H}_{37} \mathrm{O}_{1} \mathrm{~N}_{1}^{+}\right.$; calc. 319.2870).

\subsubsection{N-((Z)-3,7-Dimethylocta-2,6-dien-1-yl)adamantane-1-carboxamide 44b}

Yield 43\%. ${ }^{1} \mathrm{H}-\mathrm{NMR}\left(\mathrm{CDCl}_{3}\right)$ : 1.58 br. s (3H, 3H-21), 1.64-1.74 m (6H, 2H-4, 2H-6, 2H-10), $1.66 \mathrm{br}$. $\mathrm{s}(3 \mathrm{H}, 3 \mathrm{H}-20), 1.70$ br. s $(3 \mathrm{H}, 3 \mathrm{H}-22), 1.82 \mathrm{~d}\left(6 \mathrm{H},{ }^{3} \mathrm{~J}=3.0 \mathrm{~Hz}, 2 \mathrm{H}-2,2 \mathrm{H}-8,2 \mathrm{H}-9\right), 1.98-2.08 \mathrm{~m}(7 \mathrm{H}, \mathrm{H}-3$, $\mathrm{H}-5, \mathrm{H}-7,2 \mathrm{H}-16,2 \mathrm{H}-17), 3.78$ br. t $(2 \mathrm{H}, \mathrm{J}(13,14) \approx 7.1 \mathrm{~Hz}, 2 \mathrm{H}-13), 5.03-5.08 \mathrm{~m}(1 \mathrm{H}, \mathrm{H}-18), 5.16 \mathrm{tm}(1 \mathrm{H}$, $\mathrm{J}(14,13)=7.1 \mathrm{~Hz}, \mathrm{H}-14), 5.40$ br. s $(1 \mathrm{H}, \mathrm{NH}) .{ }^{13} \mathrm{C}-\mathrm{NMR}\left(\mathrm{CDCl}_{3}\right): 40.40$ (s, C-1), 39.15 (t, C-2, C-8, C-9), 28.03 (d, C-3, C-5, C-7), 36.42 (t, C-4, C-6, C-10), 177.61 (s, C-11), 37.06 (t, C-13), 120.87 (d, C-14), 140.01 (s, C-15), 31.85 (t, C-16), $26.46(\mathrm{t}, \mathrm{C}-17), 123.53$ (d, C-18), 131.99 (s, C-19), 25.63 (q, C-20), 17.58 (q, C-21), 23.27 (q, C-22). HR MS: $315.2551\left([M]^{+}, \mathrm{C}_{21} \mathrm{H}_{33} \mathrm{O}_{1} \mathrm{~N}_{1}{ }^{+}\right.$; calc. 315.2557).

\subsubsection{N-(3,7-Dimethyloctyl)adamantane-2-carboxamide 45a}

Yield 54\%. ${ }^{1} \mathrm{H}-\mathrm{NMR}\left(\mathrm{CDCl}_{3}\right): 0.83 \mathrm{~d}(6 \mathrm{H}, \mathrm{J}(20,19)=\mathrm{J}(21,19)=6.6 \mathrm{~Hz}, 3 \mathrm{H}-20,3 \mathrm{H}-21), 0.87 \mathrm{~d}$ $(3 \mathrm{H}, \mathrm{J}(22,15)=6.6 \mathrm{~Hz}, 3 \mathrm{H}-22), 1.05-1.13 \mathrm{~m}(3 \mathrm{H}, \mathrm{H}-16,2 \mathrm{H}-18), 1.17-1.32 \mathrm{~m}\left(4 \mathrm{H}, \mathrm{H}-14, \mathrm{H}^{\prime}-16,2 \mathrm{H}-17\right)$, $\left.1.38-1.52 \mathrm{~m} \mathrm{(3H}, \mathrm{H}^{\prime}-14, \mathrm{H}-15, \mathrm{H}-19\right), 1.56-1.61$ br. d $\left(2 \mathrm{H},{ }^{2} \mathrm{~J} \approx 12.5 \mathrm{~Hz}, \mathrm{H}-4, \mathrm{H}-9\right), 1.68-1.77 \mathrm{~m}(4 \mathrm{H}$, 2H-6, H-8, H-10), 1.79-1.83 m (1H, H-5 or H-7), 1.83-1.93 m (5H, H'-4, H-7 or H-5, H'-8, H'-9, $\left.\mathrm{H}^{\prime}-10\right)$, 2.20-2.25 m (2H, H-1, H-3), 2.41 br. s (1H, H-2), 3.21-3.34 m (2H, 2H-13), 5.55 br. s (1H, NH). ${ }^{13} \mathrm{C}-\mathrm{NMR}$ $\left(\mathrm{CDCl}_{3}\right):$ 29.88, 29.89 (2d, C-1, C-3), 49.87 (d, C-2), 33.19 (t, C-4, C-9), 27.28, 27.37 (2d, C-5, C-7), 37.26 (t, C-6), 38.25 (t, C-8, C-10), 173.81 (s, C-11), 37.32 (t, C-13), 36.76 (t, C-14), 30.59 (d, C-15), 37.00 (t, C-16), 24.54 (t, C-17), 39.07 (t, C-18), 27.81 (d, C-19), 22.46, 22.55 (2q, C-20, C-21), 19.40 (q, C-22). HR MS: $319.2873\left([M]^{+}, \mathrm{C}_{21} \mathrm{H}_{37} \mathrm{O}_{1} \mathrm{~N}_{1}{ }^{+}\right.$; calc. 319.2870).

\subsubsection{N-((Z)-3,7-Dimethylocta-2,6-dien-1-yl)adamantane-2-carboxamide 45b}

Yield 43\%. ${ }^{1} \mathrm{H}-\mathrm{NMR}\left(\mathrm{CDCl}_{3}\right): 1.57-1.62 \mathrm{~m}(2 \mathrm{H}, \mathrm{H}-4, \mathrm{H}-9), 1.58 \mathrm{~m}(3 \mathrm{H}$, all J < 1.5 Hz, 3H-21), 1.66 $\mathrm{m}(3 \mathrm{H}$, all J < $1.5 \mathrm{~Hz}, 3 \mathrm{H}-20), 1.69-1.75 \mathrm{~m}(4 \mathrm{H}, 2 \mathrm{H}-6, \mathrm{H}-8, \mathrm{H}-10), 1.70 \mathrm{~m}(3 \mathrm{H}$, all J < 2.0 Hz, 3H-22), 1.79-1.95 m (6H, H'-4, H-5, H-7, H'-8, H'-9, $\left.\mathrm{H}^{\prime}-10\right), 2.01-2.09 \mathrm{~m}(4 \mathrm{H}, 2 \mathrm{H}-16,2 \mathrm{H}-17), 2.20-2.25 \mathrm{~m}(2 \mathrm{H}$, H-1, H-3), 2.40-2.43 m (1H, H-2), 3.82-3.86 m (2H, 2H-13), 5.03-5.08 m (1H, H-18), $5.18 \mathrm{tm}(1 \mathrm{H}, \mathrm{J}(14,13)$ $=7.2 \mathrm{~Hz}, \mathrm{H}-14), 5.41$ br. s $(1 \mathrm{H}, \mathrm{NH}) .{ }^{13} \mathrm{C}-\mathrm{NMR}\left(\mathrm{CDCl}_{3}\right): 29.89$ (d, C-1, C-3), 49.89 (d, C-2), 33.19 (t, C-4, C-9), 27.29, 27.39 (2d, C-5, C-7), 37.28 (t, C-6), 38.26 (t, C-8, C-10), 173.70 (s, C-11), 36.99 (t, C-13), 121.01 
(d, C-14), 139.81 (s, C-15), 31.83 (t, C-16), 26.41 (t, C-17), 123.57 (d, C-18), 131.98 (s, C-19), 25.57 (q, C-20), 17.56 (q, C-21), 23.24 (q, C-22). HR MS: $315.2553\left([M]^{+}, \mathrm{C}_{21} \mathrm{H}_{33} \mathrm{O}_{1} \mathrm{~N}_{1}{ }^{+}\right.$; calc. 315.2557).

\subsubsection{N-(Adamantan-1-yl)-3,7-dimethyloct-6-enamide 50a}

Yield 70\%. ${ }^{1} \mathrm{H}-\mathrm{NMR}\left(\mathrm{CDCl}_{3}\right): 0.90 \mathrm{~d}(3 \mathrm{H}, \mathrm{J}(21,14)=6.6 \mathrm{~Hz}, 3 \mathrm{H}-21), 1.11-1.20 \mathrm{~m}(1 \mathrm{H}, \mathrm{H}-15)$, 1.29-1.37 m (1H, H'-15), 1.57 br. s (3H, 3H-20), 1.61-1.68 m (9H, 2H-4, 2H-6, 2H-10, 3H-19), $1.81 \mathrm{dd}(1 \mathrm{H}$, $\left.{ }^{2} \mathrm{~J}=13.6 \mathrm{~Hz}, \mathrm{~J}(13,14)=8.4 \mathrm{~Hz}, \mathrm{H}-13\right), 1.87-2.02 \mathrm{~m}(9 \mathrm{H}, \mathrm{H}-14,2 \mathrm{H}-16,2 \mathrm{H}-2,2 \mathrm{H}-8,2 \mathrm{H}-9), 2.02-2.06 \mathrm{~m}$ $(3 \mathrm{H}, \mathrm{H}-3, \mathrm{H}-5, \mathrm{H}-7), 2.09 \mathrm{dd}\left(1 \mathrm{H},{ }^{2} \mathrm{~J}=13.6 \mathrm{~Hz}, \mathrm{~J}\left(13^{\prime}, 14\right)=5.9 \mathrm{~Hz}, \mathrm{H}^{\prime}-13\right), 5.06 \mathrm{tm}(1 \mathrm{H}, \mathrm{J}(17,16)=7.2 \mathrm{~Hz}$, H-17), 5.20 br. s (1H, H-11). ${ }^{13} \mathrm{C}-\mathrm{NMR}\left(\mathrm{CDCl}_{3}\right): 51.81$ (s, C-1), 41.56 (t, C-2, C-8, C-9), 29.32 (d, C-3, C-5, C-7), 36.24 (t, C-4, C-6, C-10), 171.79 (s, C-12), 45.35 (t, C-13), 30.46 (d, C-14), 36.73 (t, C-15), 25.34 (t, C-16), 124.33 (d, C-17), 131.29 (s, C-18), 25.58 (q, C-19), 17.54 (q, C-20), 19.31 (q, C-21). HR MS: 303.2558 $\left([M]^{+}, \mathrm{C}_{20} \mathrm{H}_{33} \mathrm{O}_{1} \mathrm{~N}_{1}^{+}\right.$; calc. 303.2557).

\subsubsection{N-(Adamantan-2-yl)-3,7-dimethyloct-6-enamide 50b}

Yield $72 \% .{ }^{1} \mathrm{H}-\mathrm{NMR}\left(\mathrm{CDCl}_{3}\right): 0.92 \mathrm{~d}(3 \mathrm{H}, \mathrm{J}(21,14)=6.6 \mathrm{~Hz}, 3 \mathrm{H}-21), 1.15-1.22 \mathrm{~m}(1 \mathrm{H}, \mathrm{H}-15)$, 1.32-1.39 m (1H, $\left.\mathrm{H}^{\prime}-15\right), 1.57$ br. s $(3 \mathrm{H}, 3 \mathrm{H}-20), 1.60-1.65 \mathrm{~m}(2 \mathrm{H}, \mathrm{H}-4, \mathrm{H}-9), 1.65 \mathrm{~m}(3 \mathrm{H}$, all J < 2.0 Hz, $3 \mathrm{H}-19), 1.70-1.76 \mathrm{~m}\left(4 \mathrm{H}, \mathrm{H}^{\prime}-4,2 \mathrm{H}-6, \mathrm{H}^{\prime}-9\right), 1.77-1.85 \mathrm{~m}(6 \mathrm{H}, \mathrm{H}-5, \mathrm{H}-7,2 \mathrm{H}-8,2 \mathrm{H}-10), 1.86-1.90 \mathrm{~m}(2 \mathrm{H}$, $\mathrm{H}-1, \mathrm{H}-3), 1.90-2.05 \mathrm{~m}(4 \mathrm{H}, \mathrm{H}-13, \mathrm{H}-14,2 \mathrm{H}-16), 2.15-2.22 \mathrm{~m}\left(1 \mathrm{H}, \mathrm{H}^{\prime}-13\right), 4.03-4.06 \mathrm{~m}(1 \mathrm{H}, \mathrm{H}-2), 5.06 \mathrm{tm}$ $(1 \mathrm{H}, \mathrm{J}(17,16)=7.2 \mathrm{~Hz}, \mathrm{H}-17), 5.70$ br. d $(1 \mathrm{H}, \mathrm{J}(11,2) \approx 8.0 \mathrm{~Hz}, \mathrm{NH}) .{ }^{13} \mathrm{C}-\mathrm{NMR}\left(\mathrm{CDCl}_{3}\right): 31.82,31.86(2 \mathrm{~d}$, C-1, C-3), 52.90 (d, C-2), 31.85 (t, C-4, C-9), 27.01, 27.12 (2d, C-5, C-7), 37.43 (t, C-6), 36.98, 37.01 (2t, C-8, C-10), 171.41 (s, C-12), 44.87 (t, C-13), 30.55 (d, C-14), 36.79 (t, C-15), 25.34 (t, C-16), 124.26 (d, C-17), 131.37 (s, C-18), 25.58 (q, C-19), 17.54 (q, C-20), 19.41 (q, C-21). HR MS: $303.2562\left([M]^{+}, \mathrm{C}_{20} \mathrm{H}_{33} \mathrm{O}_{1} \mathrm{~N}_{1}{ }^{+}\right.$; calc. 303.2557).

\subsubsection{General Procedure for Thioamides $46 \mathbf{a}, \mathbf{b} ; \mathbf{4 7 a}, \mathbf{b} ; 51$}

To the solution of amides $44 \mathbf{a}, \mathbf{b} ; \mathbf{4 5 a}, \mathbf{b}$ (53 mg, $0.16 \mathrm{mmol}, 1 \mathrm{eq}), 50 \mathbf{b}(50 \mathrm{mg}, 0.16 \mathrm{mmol}, 1 \mathrm{eq})$ in dry toluene $(10 \mathrm{~mL})$, Py $(6.6 \mu \mathrm{L}, 82 \mu \mathrm{mol}, 0.5 \mathrm{eq})$ and Lawesson's reagent $(0.20,0.49 \mathrm{mmol}, 3 \mathrm{eq})$ were added, the mixture was refluxed overnight in argon atmosphere [52]. The solvent was evaporated, the residue was dissolved in $\mathrm{Et}_{2} \mathrm{O}$ and washed with saturated solution of $\mathrm{NaHCO}_{3}(20 \mathrm{~mL})$ and brine $(20 \mathrm{~mL})$ consequentially and dried with $\mathrm{Na}_{2} \mathrm{SO}_{4}$. Thioamides were purified by column chromatography.

\subsubsection{N-(3,7-Dimethyloctyl)adamantane-1-carbothioamide 46a}

Yield $88 \% .{ }^{1} \mathrm{H}-\mathrm{NMR}\left(\mathrm{CDCl}_{3}\right): 0.84 \mathrm{~d}(6 \mathrm{H}, \mathrm{J}(20,19)=\mathrm{J}(21,19)=6.6 \mathrm{~Hz}, 3 \mathrm{H}-20,3 \mathrm{H}-21), 0.91 \mathrm{~d}(3 \mathrm{H}$, $\mathrm{J}(22,15)=6.5 \mathrm{~Hz}, 3 \mathrm{H}-22), 1.07-1.17 \mathrm{~m}(3 \mathrm{H}, \mathrm{H}-16,2 \mathrm{H}-18), 1.18-1.32 \mathrm{~m}\left(3 \mathrm{H}, \mathrm{H}^{\prime}-16,2 \mathrm{H}-17\right), 1.40-1.54 \mathrm{~m}$ (3H, H-14, H-15, H19), 1.61-1.74 m (7H, 2H-4, 2H-6, 2H-10, H'-14), 1.94 d (6H, ${ }^{3} \mathrm{~J}=3.0 \mathrm{~Hz}, 2 \mathrm{H}-2,2 \mathrm{H}-8$, 2H-9), 2.04-2.09 m (3H, H-3, H-5, H-7), 3.60-3.71 m (2H, 2H-13). ${ }^{13} \mathrm{C}-\mathrm{NMR}\left(\mathrm{CDCl}_{3}\right): 45.79$ (s, C-1), 41.74 (t, C-2, C-8, C-9), 28.49 (d, C-3, C-5, C-7), 36.30 (t, C-4, C-6, C-10), 212.60 (s, C-11), 44.32 (t, C-13), 34.92 (t, C-14), 30.88 (d, C-15), 36.92 (t, C-16), 24.52 (t, C-17), 39.04 (t, C-18), 27.81 (d, C-19), 22.45, 22.55 (2q, C-20, C-21), 19.54 (q, C-22). HR MS: $335.2638\left([M]^{+}, \mathrm{C}_{21} \mathrm{H}_{37} \mathrm{~N}_{1} \mathrm{~S}_{1}{ }^{+}\right.$; calc. 335.2641).

\subsubsection{N-((Z)-3,7-Dimethylocta-2,6-dien-1-yl)adamantane-1-carbothioamide 46b}

Yield 31\%. A NMR spectra of trans- and cis-isomers at C-14-C-15 bond was obtained for their mixture in ratio $\approx 1.0: 0.8$, respectively. In ${ }^{1} \mathrm{H}-\mathrm{NMR}$ spectrum the signals of corresponding protons of isomers superimpose on each other. In ${ }^{13} \mathrm{C}-\mathrm{NMR}$ spectrum, most of the carbon signals of both isomers differ. ${ }^{1} \mathrm{H}-\mathrm{NMR}\left(\mathrm{CDCl}_{3}\right)$ : $1.57 \mathrm{br}$. s (3H-21 of both isomers), $1.63-1.74 \mathrm{~m}(2 \mathrm{H}-4,2 \mathrm{H}-6,2 \mathrm{H}-10$ of both isomers), $1.65 \mathrm{~m}$ (3H-20 of both isomers, $3 \mathrm{H}-22$ of trans-isomer), $1.72 \mathrm{~m}$ (3H-22 of cis-isomer), 1.92-1.95 m (2H-2, 2H-8, 2H-9 of both isomers), 1.98-2.12 m (H-3, H-5, H-7, 2H-16, 2H-17 of both isomers), $4.14-4.19 \mathrm{~m}$ (2H-13 of both isomers), $5.01-5.06 \mathrm{~m}$ (H-18 of both isomers), $5.26-5.31 \mathrm{~m}$ (H-14 of both isomers), 7.09 br. s (NH of both isomers). ${ }^{13} \mathrm{C}-\mathrm{NMR}$ of trans-isomer $\left(\mathrm{CDCl}_{3}\right): 45.69$ (s, C-1), 41.69 
(t, C-2, C-8, C-9), 28.45 (d, C-3, C-5), 36.25 (t, C-4, C-6, C-10), 212.19 (s, C-11), 44.35 (t, C-13), 117.84 (d, C-14), 142.31 (s, C-15), 39.27 (t, C-16), 26.10 (t, C-17), 123.48 (d, C-18), 131.71 (s, C-19), 25.52 (q, C-20), 17.56 (q, C-21), 16.38 (q, C-22). ${ }^{13} \mathrm{C}-\mathrm{NMR}$ of cis-isomer $\left(\mathrm{CDCl}_{3}\right)$ : 45.68 (s, C-1), 41.69 (t, C-2, C-8, C-9), 28.45 (d, C-3, C-5, C-7), 36.25 (t, C-4, C-6, C-10), 212.22 (s, C-11), 44.13 (t, C-13), 118.57 (d, C-14), 142.52 (s, C-15), 31.96 (t, C-16), 26.39 (t, C-17), 123.24 (d, C-18), 132.14 (s, C-19), 25.56 (q, C-20), 17.58 (q, C-21), 23.25 (q, C-22). HR MS: $330.2249\left([M]^{+}, \mathrm{C}_{21} \mathrm{H}_{33} \mathrm{~N}_{1} \mathrm{~S}_{1}{ }^{+}\right.$; calc. 331.2328).

\subsubsection{N-(3,7-Dimethyloctyl)adamantane-2-carbothioamide 47a}

Yield $87 \% .{ }^{1} \mathrm{H}-\mathrm{NMR}\left(\mathrm{CDCl}_{3}\right): 0.83 \mathrm{~d}(6 \mathrm{H}, \mathrm{J}(20,19)=\mathrm{J}(21,19)=6.6 \mathrm{~Hz}, 3 \mathrm{H}-20,3 \mathrm{H}-21), 0.91 \mathrm{~d}(3 \mathrm{H}$, $\mathrm{J}(22,15)=6.5 \mathrm{~Hz}, 3 \mathrm{H}-22), 1.07-1.17 \mathrm{~m}(3 \mathrm{H}, \mathrm{H}-16,2 \mathrm{H}-18), 1.18-1.33 \mathrm{~m}\left(3 \mathrm{H}, \mathrm{H}^{\prime}-16,2 \mathrm{H}-17\right), 1.41-1.53 \mathrm{~m}$ (3H, H-14, H-15, H-19), 1.56-1.62 m (2H, H-4, H-9), 1.63-1.70 m (1H, H'-14), 1.70-1.73 m (2H, 2H-6), 1.76-1.94 m (8H, H'-4, H-5, H-7, 2H-8, H'-9, 2H-10), 2.60-2.63 m (2H, H-1, H-3), 2.65-2.69 m (1H, H-2), 3.67-3.79 m (2H, H-13), 7.34 br. s $(1 \mathrm{H}, \mathrm{NH}) .{ }^{13} \mathrm{C}-\mathrm{NMR}\left(\mathrm{CDCl}_{3}\right): 31.41,31.42(2 \mathrm{~d}, \mathrm{C}-1, \mathrm{C}-3), 56.15$ (d, C-2), 32.56 (t, C-4, C-9), 27.11, 27.25 (2d, C-5, C-7), 37.09 (t, C-6), 38.40 (t, C-8, C-10), 206.53 (s, C-11), 44.32 (t, C-13), 35.00 (t, C-14), 30.85 (d, C-15), 36.89 (t, C-16), 24.53 (t, C-17), 39.01 (t, C-18), 27.80 (d, C-19), 22.44, 22.54 (2q, C-20, C-21), 19.47 (q, C-22). HR MS: $335.2637\left([M]^{+}, \mathrm{C}_{21} \mathrm{H}_{37} \mathrm{~N}_{1} \mathrm{~S}_{1}{ }^{+}\right.$; calc. 335.2641).

\subsubsection{N-((Z)-3,7-Dimethylocta-2,6-dien-1-yl)adamantane-2-carbothioamide 47b}

Yield $60 \% .{ }^{1} \mathrm{H}-\mathrm{NMR}\left(\mathrm{CDCl}_{3}\right): 1.57-1.62 \mathrm{~m}(2 \mathrm{H}, \mathrm{H}-4, \mathrm{H}-9), 1.58$ br. s $(3 \mathrm{H}, 3 \mathrm{H}-21), 1.66 \mathrm{~m}(3 \mathrm{H}$, all $\mathrm{J}<$ $1.5 \mathrm{~Hz}, 3 \mathrm{H}-20), 1.70-1.73 \mathrm{~m}(2 \mathrm{H}, 2 \mathrm{H}-6), 1.75 \mathrm{~m}(3 \mathrm{H}$, all J < 2.0 Hz, 3H-22), 1.76-1.95 m (8H, H'-4, H-5, H-7, 2H-8, 2H-10, H'-9), 2.02-2.12 m (4H, 2H-16, 2H-17), 2.60-2.63 m (2H, H-1, H-3), 2.65-2.68 m (1H, $\mathrm{H}-2), 4.23-4.27 \mathrm{~m}(2 \mathrm{H}, 2 \mathrm{H}-13), 5.03-5.08 \mathrm{~m}(1 \mathrm{H}, \mathrm{H}-18), 5.31 \mathrm{tm}(1 \mathrm{H}, \mathrm{J}(14,13)=7.1 \mathrm{~Hz}, \mathrm{H}-14), 7.17 \mathrm{br} . \mathrm{s}$ $(1 \mathrm{H}, \mathrm{NH}) .{ }^{13} \mathrm{C}-\mathrm{NMR}\left(\mathrm{CDCl}_{3}\right): 31.42(\mathrm{~d}, \mathrm{C}-1, \mathrm{C}-3), 56.06(\mathrm{~d}, \mathrm{C}-2), 32.59(\mathrm{t}, \mathrm{C}-4, \mathrm{C}-9), 27.14,27.29(2 \mathrm{~d}, \mathrm{C}-5$, C-7), 37.15 (t, C-6), 38.43 (t, C-8, C-10), 206.22 (s, C-11), 44.22 (t, C-13), 118.56 (d, C-14), 142.59 (s, C-15), 32.03 (t, C-16), 26.42 (t, C-17), 123.29 (d, C-18), 132.27 (s, C-19), 25.57 (q, C-20), 17.60 (q, C-21), 23.30 (q, C-22). HR MS: $330.2245\left([M]^{+}, \mathrm{C}_{21} \mathrm{H}_{33} \mathrm{~N}_{1} \mathrm{~S}_{1}{ }^{+}\right.$; calc. 331.2328).

\subsubsection{N-(Adamantan-2-yl)-3,7-dimethyloct-6-enethioamide 51}

Yield 64\%. ${ }^{1} \mathrm{H}-\mathrm{NMR}\left(\mathrm{CDCl}_{3}\right): 0.90 \mathrm{~d}(3 \mathrm{H}, \mathrm{J}(21,14)=6.7 \mathrm{~Hz}, 3 \mathrm{H}-21), 1.13-1.21 \mathrm{~m}(1 \mathrm{H}, \mathrm{H}-15)$, $1.33-1.40 \mathrm{~m}\left(1 \mathrm{H}, \mathrm{H}^{\prime}-15\right), 1.56$ br. s $(3 \mathrm{H}, 3 \mathrm{H}-20), 1.64$ br. s (3H, 3H-19), $1.68-1.72 \mathrm{~m}(4 \mathrm{H}, 2 \mathrm{H}-9,2 \mathrm{H}-10)$, $1.72-1.76 \mathrm{~m}(2 \mathrm{H}, 2 \mathrm{H}-6), 1.82-1.85 \mathrm{~m}(4 \mathrm{H}, 2 \mathrm{H}-4,2 \mathrm{H}-8), 1.85-1.89 \mathrm{~m}(2 \mathrm{H}, \mathrm{H}-5, \mathrm{H}-7), 1.91-2.05 \mathrm{~m}(2 \mathrm{H}$, 2H-16), 2.08-2.16 m (3H, H-1, H-3, H-14), $2.37 \mathrm{dd}\left(1 \mathrm{H},{ }^{2} \mathrm{~J}=13.1 \mathrm{~Hz}, \mathrm{~J}(13,14)=8.4 \mathrm{~Hz}, \mathrm{H}-13\right), 2.67 \mathrm{dd}$ $\left(1 \mathrm{H},{ }^{2} \mathrm{~J}=13.1 \mathrm{~Hz}, \mathrm{~J}\left(13^{\prime}, 14\right)=6.2 \mathrm{~Hz}, \mathrm{H}^{\prime}-13\right), 4.56 \mathrm{ddd}(1 \mathrm{H}, \mathrm{J}(2, \mathrm{NH})=8 \mathrm{~Hz}, \mathrm{~J}(2,1) \approx \mathrm{J}(2,3) \approx 3.0 \mathrm{~Hz}$, $\mathrm{H}-2), 5.05 \mathrm{tm}(1 \mathrm{H}, \mathrm{J}(17,16)=7.1 \mathrm{~Hz}$, other $\mathrm{J}<2.0 \mathrm{~Hz}, \mathrm{H}-17), 7.40$ br. d $(1 \mathrm{H}, \mathrm{J}(\mathrm{NH}, 2)=8 \mathrm{~Hz}, \mathrm{NH})$. ${ }^{13} \mathrm{C}-\mathrm{NMR}\left(\mathrm{CDCl}_{3}\right): 30.47,30.49$ (2d, C-1, C-3), 58.84 (d, C-2), 36.67, 36.71 (2t, C-4, C-8), 26.89 (d, C-5, C-7), 37.20 (t, C-6), 32.24 (t, C-9, C-10), 202.97 (s, C-12), 55.52 (t, C-13), 33.45 (d, C-14), 36.34 (t, C-15), 25.28 (t, C-16), 124.09 (d, C-17), 131.43 (s, C-18), 25.55 (q, C-19), 17.53 (q, C-20), 18.75 (q, C-21). HR MS: $319.2327\left([M]^{+}, \mathrm{C}_{21} \mathrm{H}_{33} \mathrm{~N}_{1} \mathrm{~S}_{1}{ }^{+}\right.$; calc. 319.2328).

\subsection{Modelling and Screening}

The compounds were docked against the crystal structure of Tdp1 PDB ID: 1MU7, resolution $2.0 \AA$ Å) [53] which was obtained from the Protein Data Bank (PDB, Cambridge, UK) [54,55]. The Scigress version FJ 2.6 program [56] was used to prepare the crystal structure for docking (i.e., the hydrogen atoms were added, the co-crystallised tungsten(VI)ion was removed as well as crystallographic water molecules). For further studies, HOH631 and HOH832 water molecules were kept in the crystal structure. The Scigress software suite was also used to build the inhibitors and the MM2 [57] force field was used to optimise the structures. The docking centre was defined as the position of the hydrogen atom of His263, whereby nitrogen formed a coordination bond with the tungsten ion $(x=7.319$, $\mathrm{y}=0.055, \mathrm{z}=96.688$ ) with 10 A radius. Fifty docking runs were allowed for each ligand with default search efficiency $(100 \%)$. The basic amino acids lysine and arginine were defined as protonated. 
Furthermore, aspartic and glutamic acids were assumed deprotonated. The GoldScore(GS) [58] and ChemScore(CS) [59,60], Piecewise Linear Potential (ChemPLP) [61] and Astex Statistical Potential (ASP) [62] scoring functions were implemented to validate the predicted binding modes and relative energies of the ligands using the GOLD v5.4.1 software suite. The QikProp 3.2 [63] software package was used to calculate the molecular descriptors of the molecules. The reliability of it is established for the calculated descriptors [64].

\subsection{Biological Study}

\subsubsection{TDP1 Activity}

A fluorophore quencher-coupled DNA-biosensor for real-time measurement of Tdp1 cleavage activity was designed recently in our laboratory [29]. This biosensor is a short single-stranded oligonucleotide with a 5'-FAM fluorophore and a 3'-BHQ1 (black hole quencher 1) quencher.

Recombinant protein Tdp1 was expressed in Escherichia coli (pET 16B plasmid containing Tdp1 cDNA was provided by Dr. K.W. Caldecott, University of Sussex, United Kingdom) and isolated as described $[7,65]$. The reaction mixture in a final volume $200 \mu \mathrm{L}$ contained Tdp1 reaction buffer $(50 \mathrm{mM}$ Tris- $\mathrm{HCl}, 50 \mathrm{mM} \mathrm{NaCl}, 7 \mathrm{mM} \beta$-mercaptoethanol), $50 \mathrm{nM}$ biosensor and varied concentrations of potential inhibitor. Purified Tdp1 was added in a final concentration of $1.5 \mathrm{nM}$.

The reaction mixtures were incubated at a temperature of $26{ }^{\circ} \mathrm{C}$ in a POLARstar OPTIMA fluorimeter, BMG LABTECH, GmbH, Germany, to measure fluorescence intensity every $1 \mathrm{~min}$ $(\mathrm{Ex} 485 / \mathrm{Em} 520 \mathrm{~nm})$. Tdp1 inhibition was calculated by comparing the rate of increase in fluorescence of biosensor in the presence of compound to that of DMSO (1.5\%) control wells. The measurements were carried out in at least two independent experiments. $\mathrm{IC}_{50}$ values were determined using a six-point concentration response curve. The data were imported into MARS Data Analysis 2.0 program (BMG $\mathrm{LABTECH}$ ) and the slope during the linear phase (here data from 0 to $7 \mathrm{~min}$ ) was calculated.

\subsubsection{Cytotoxicity Assay}

Analysis of the toxicity of the compounds was examined against human cell line A-549 (adenocarcinomic human alveolar basal epithelial cells) using a standard MTT (3-(4,5-dimethylthiazol2-yl)-2,5-diphenyl-2H-tetrazolium bromide) test [66] by colorimetric measurement of the amount of formazan converted from MTT in cells exposed to the compounds. The cell line was obtained from the Russian Cell Culture Collection (RCCC) Institute of Cytology RAS, St. Petersburg, Russia. The cells were grown in IMDM medium, with $40 \mu \mathrm{g} / \mathrm{mL}$ gentamicin, $50 \mathrm{IU} / \mathrm{mL}$ penicillin, $50 \mu \mathrm{g} / \mathrm{mL}$ streptomycin (MP Biomedicals, Solon, USA) and in the presence of $10 \%$ fetal bovine serum (Biolot, Saint Petersburg, Russia) in $5 \% \mathrm{CO}_{2}$ atmosphere. After formation of a 30-50\% monolayer, tested compounds were added to the medium (the volume of added reagents was $1 / 100$ of the total volume of the culture medium, the amount of DMSO was $1 \%$ of the final volume), and the cell culture was monitored for 3 days. Control cells were grown in the presence of $1 \%$ DMSO. The Tdp1 inhibitor concentrations ranged from 1 to $100 \mu \mathrm{M}$. Topotecan concentrations ranged from 30 to $3000 \mathrm{nM}$ in the presence of $5 \mu \mathrm{M}$ Tdp 1 inhibitor or $1 \%$ DMSO. The measurements were carried out in three parallel experiments.

\subsubsection{Binding Studies}

Intrinsic protein fluorescence assay were conducted according to the protocol as described in references $[35,45,49]$. The concentrations of recombinant Tdp1 and ligand were $10 \mu \mathrm{M}$ and $1 \mathrm{mM}$, respectively. Excitation wavelength was $280 \mathrm{~nm}$ and intrinsic fluorescence was measured between 300 and $450 \mathrm{~nm}$.

Thermal shift assay was carried out using a modified protocol that was described in [67]. Experiments were carried out using an Applied BioSystems QuantStudio 3 Real-Time PCR system. Samples were measured using 96-well PCR plates (Bio-Rad). Reaction volume was $25 \mu \mathrm{L}$. Protein unfolding was monitored by measuring the fluorescence of the SYPRO Orange dye (Sigma Aldrich, St 
Louis, MO, US). Temperature was increased from 25 to $95^{\circ} \mathrm{C}$ at $1{ }^{\circ} \mathrm{C}$ increments every $60 \mathrm{~s}$. The final concentrations of Tdp1 and compounds were 20 and $100 \mu \mathrm{M}$, respectively. Buffer contained $20 \mathrm{mM}$ Tris ( $\mathrm{pH} \mathrm{8.0),} 250 \mathrm{mM} \mathrm{NaCl}$ and $2.5 \times$ SYPRO Orange dye. Measurements were performed in triplicate. For determination of melting temperature values, melting curve for each data set was analysed by SigmaPlot 13 (USA) and fitted with the Sigmoid, 3-parameter model.

\section{Results and Discussion}

\subsection{Organic Synthesis}

To synthetize amides and thioamides, commercially available 1-adamantane carboxylic acid chloride 31 was used. An isomeric chloride of 2-adamantane carboxylic acid 32 was obtained starting from adamantan-2-one 33 according to previously published methods [68-70] including the synthesis of epoxide 34, its treatment with boron trifluoride etherate and subsequent oxidization (Scheme 1). 2-Adamantane carboxylic acid 35 was refluxed in thionyl chloride yielding chloride 32 quantitatively. Monoterpene amines 36 and 37 were obtained by Gabriel synthesis. Using this approach, bromides $\mathbf{4 0}$ and $\mathbf{4 1}$ were synthesized starting from 3,7-dimethyloctanol 38 and nerol 39, respectively [42,51]. Then the bromides were stirred with potassium phthalimide at $60{ }^{\circ} \mathrm{C}$ giving compounds 42 and 43 . Compounds 40 and 41 were purified by column chromatography. Their interaction with ethylenediamine led to amines 37 and 38. It was found that subsequent dissolving in hexane and precipitate filtration followed by solvent evaporation led to pure amines (Scheme 1).

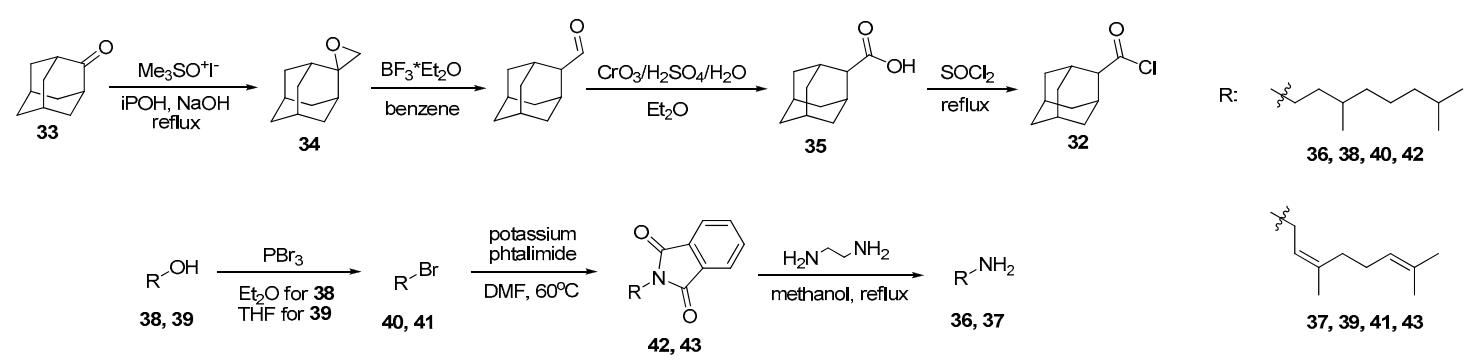

Scheme 1. Synthesis of 2-adamantane carboxylic acid chloride 32 and monoterpene amines 42 and 43.

By interacting chlorides 31 and 32 with amines 36 and 37 a set of amides $44 a, b ; 45 a, b$ containing acyclic monoterpenes moieties was synthesized (Scheme 2). The interaction of amides $44 \mathbf{a}, \mathbf{b} ; \mathbf{4 5} \mathbf{a}, \mathbf{b}$ with Lawesson's reagent led to thioamides $46 \mathbf{a}, \mathbf{b} ; \mathbf{4 7 a}, \mathbf{b}$. For derivative $46 \mathrm{~b}$, the isomerization of nerol fragment during reflux was observed and the resulting mixture contained both citral derivatives in a trans- and cis-isomers ratio of 1.0:0.8. For structure activity relationship (SAR) studies, the synthesis of citronellic acid derivatives containing adamantane fragments was performed. Derivative 48 was obtained by refluxing citronellic acid with thionyl chloride. Interaction of 48 with 1- and 2-adamnataneamines $\mathbf{4 9} \mathbf{a}, \mathbf{b}$ in the presence of trimethylamine led to amides $\mathbf{5 0} \mathbf{a}, \mathbf{b}$ (Scheme 2). Synthesis of citronellic acid thioamide 51 containing 2-adamantane amine residue was performed by reflux with Lawesson's reagent. 


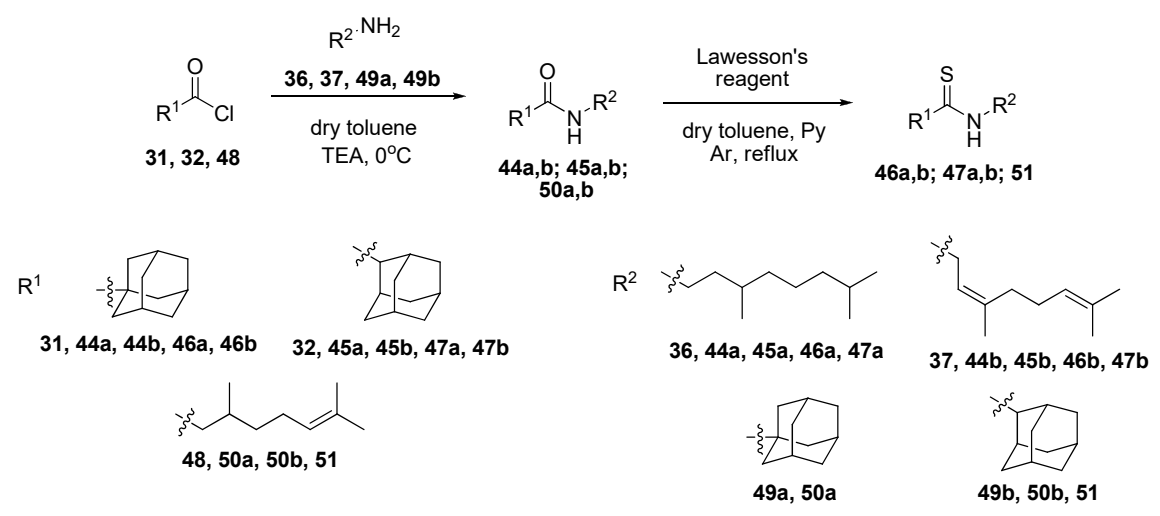

Scheme 2. The synthesis of amides $44 a, b ; 45 a, b ; 50 a, b$ and thioamides $46 a, b ; 47 a, b ; 51$ of $1-, 2-$ adamantane carboxylic acid and citronellic acid.

\subsection{Inhibition Studies}

The binding and inhibition potency of the compounds were tested against purified recombinant Tdp1. Binding experiments were conducted using thermal shift assay [71] and an intrinsic fluorescence binding assay that we previously developed $[35,45,49]$. Thermal shift assay measures the stability of proteins against thermal degradation. If a molecule binds to the protein, it may be stabilized or destabilized by the binding of the molecule, thus leading to a shift of the protein melting temperature. The intrinsic fluorescence binding assay measures the fluorescence intensity of tryptophan residues of a protein. The active site of Tdp1 is close to several tryptophan residues. Binding of Tdp1 inhibitors may change the environment of these tryptophan residues, thus leading to quenching of fluorescence intensity. However, no significant changes in the melting temperature of Tdp1 or quenching of Tdp1 fluorescence intensity were observed (Supplementary Figures S1-S6). This may be due to the low solubility of our compounds ( $1 \mathrm{mM}$ or less) in aqueous buffer. As our binding assays typically required at least tens of $\mu \mathrm{M}$ of Tdp1, we reasoned that the lack of changes in melting temperature and intrinsic fluorescence intensity are likely due the low ligand-to-protein ratio needed in our experiments, which did not allow complete formation of Tdp1-inhibitor complex.

Therefore, the inhibition potency of our compounds $44 a, b-47 a, b ; 50 a, b ; 51$ were tested against Tdp1 by using a biosensor assay previously developed [29]. The biosensor is 16-mer single-stranded oligonucleotide containing a 5'-FAM (5(6)-carboxyfluorescein) fluorophore donor and a quenching 3'-BHQ1 (Black Hole Quencher-1). Because BHQ1 and FAM are located within the Förster radius, the fluorescence is quenched. The mechanism of action is based on the Tdp1 enzyme's ability to selectively cleave substituents from the 3 '-end of DNA [72], thus deriving the Tdp1 activity by real-time fluorescence detection $[29,32]$. Table 1 shows the Tdp1 inhibition potency of the compounds $\mathbf{4 4 a , b - 4 7 a , b ; 5 0 a , b ; 5 1 . ~ F u r a m i d i n e ~ 1 1 , ~ a ~ c o m m e r c i a l l y - a v a i l a b l e ~ T d p 1 ~ i n h i b i t o r , ~ w a s ~ u s e d ~ a s ~ a ~ p o s i t i v e ~}$ control. Compounds $46 \mathrm{~b}$ and $\mathbf{4 7 b}$ were not investigated in Tdp1 inhibitory activity tests due to their instability. The compounds synthesized can be divided in to two groups: (1) amides and thioamides of 1- and 2-adamantane carboxylic acids (44a,b; $45 \mathbf{a}, \mathbf{b} ; 46 \mathbf{a} ; 47 \mathbf{a})$; (2) derivatives of citronellic acid $(\mathbf{5 0 a}, \mathbf{b} ; \mathbf{5 1})$. Comparing inhibitory activity of the first group (Table 1) and compounds synthesized previously [49] it can be concluded that amides $\mathbf{4 4 - 4 7}$ are less active than their ester counterparts are. For example, in the cases of derivatives 29 (ester), 44 (amide), 48 (thioamide), which are structural analogues, the following $\mathrm{IC}_{50}$ values were obtained: $0.92,>75$ and $3.3 \mu \mathrm{M}$, respectively. Note that derivatives of 2-adamantane carboxylic acid were found to be more active compared to compounds obtained from 1-admantane carboxylic acid. Thus, amides $44 \mathbf{a}$ and $\mathbf{4 4 b}$ were not active, whilst $\mathbf{4 5 a}$ and $45 \mathrm{~b}$ showed Tdp $150 \%$ inhibition at 5.2 and $2.5 \mu \mathrm{M}$, respectively (Table 1). Comparing amide 45a and thioamide 47 a activity it can be concluded that, for 2-adamantane carboxylic acid derivatives, oxygen atom replacement by sulphur also led to activity increasing, with $\mathrm{IC}_{50}$ being $5.2 \mu \mathrm{M}$ for $45 \mathrm{a}$ and $0.64 \mu \mathrm{M}$ for $47 \mathbf{a}$. 
Similar trends were observed for citronellic acid derivatives $\mathbf{5 0 a}, \mathbf{b}$; $\mathbf{5 1 .}$ For example, 1-aminoadamnatane derivative 50a was not active whilst 2-aminoadamantane $50 \mathrm{~b}$ activity was found to be slightly higher and the best $\mathrm{IC}_{50}$ value was obtained for thioamide $51(2.3 \mu \mathrm{M})$, also a 2-aminoadamantane derivative.

Table 1. Tdp1 inhibition potency of the adamantine derivatives.

(44a

* $95 \%$ confidence intervals are given in parentheses.

In general, based on the results shown in Table 1 and previously published results [49], the following conclusions can be made: (1) Thioamides are more active than the esters and they have better activity than their amide counterparts; (2) 2-adamanatne substituted derivatives (45a, 45b, 47a, $50 \mathrm{~b}$ and 51 ) are more active compared to 1 -adamantane substituted isomers (44a, 44b, 46a and 50a).

\subsection{Cytotoxicity}

The intrinsic cytotoxicity of the adamantane derivatives was examined using the lung adenocarcinoma A-549 cell line and the standard MTT assay, the results are shown in Figure S16. In general, all the compounds were mildly toxic or non-cytotoxic: $\mathrm{CC}_{50}$ values (cytotoxic concentration at $50 \%$ ) were $>80 \mu \mathrm{M}$. Since Tdp 1 inhibitors are supposed to be used for adjuvant therapy with established antitumor drugs, it is important that the Tdp1 therapeutic agents do not cause any side effects.

As already mentioned, the combined use of TOP1 poisons and Tdp1 inhibitors can lead to a synergistic effect. Topotecan is a bioavailable derivative of CPT, used in the clinic to treat, for example, 
lung cancer [73]. Despite the important role of topotecan in cancer therapy, resistance to CPTs and severe side effects are reported [73]. One of the ways to overcome resistance and/or reduce the dose of topotecan, and consequently its toxicity, is the joint use with Tdp1 inhibitors.

The cytotoxic effect of topotecan was studied in conjunction with the inhibitors. The adamantane derivatives were used in the nontoxic concentration of $5 \mu \mathrm{M}$. Most compounds had a weak or no sensitizing effect (data not shown). The most pronounced was by compound 46a (Figure 3), thioamide of 2-adamantane carboxylic acid with 3,7-dimethyloctane fragment.

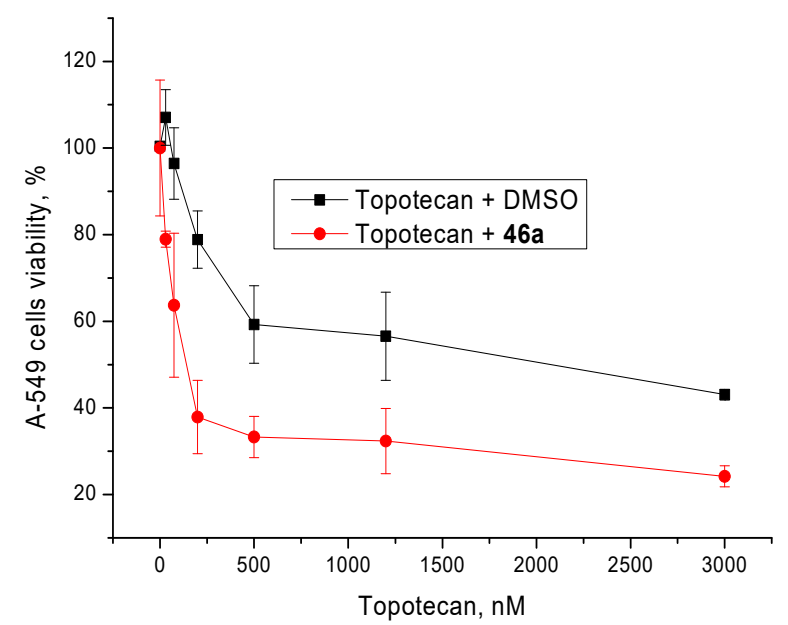

Figure 3. Dose-dependent action of topotecan in combination with $5 \mu \mathrm{M}$ of $46 \mathrm{a}$ on A- 549 cells viability.

Therefore, 46a is the most promising derivative as it has high enough anti-Tdp1 activity, low cytotoxicity and synergism with topotecan.

\subsection{Molecular Modeling}

The eleven molecules were docked into the binding site of Tdp1 (PDB ID: 1MU7, resolution $2.0 \AA$ ) [53] with reasonable scores, as shown in Table S1 in the supplementary materials. Furthermore, two water molecules (H2O631 and H2O832) were kept in the binding pocket and all the ligands docked. The modelling shows that all the ligands show a similar plausible binding mode for both scenarios. The ligands have higher scores with water (see Table S1), which suggests that they contribute to the stability of the protein and binding. Indeed, the modelling predicts that the ligands interact with the water molecules. For the most interesting compound 46a, the sulphur atom forms an H-bond with HOH631, this water molecule also interacts with Thr261, forming an H-bond bridge between the ligand the protein. The second water molecule HOH832 does not form direct H-bonds with the ligand but nudges the adamantane moiety closer to a lipophilic region. The adamantane moiety occupies a relatively lipophilic portion of the binding pocket whilst the thioamide occupies a hydrophilic portion as shown with Figure $4 \mathrm{~A}$.

The calculated molecular descriptors molecular weight (MW), number of hydrogen bond donors (HDs), number of hydrogen bond acceptors (HAs), lipophilicity (Log P), polar surface area (PSA) and number of rotatable bonds (RBs) are given in Table S2 in the supplementary materials. HDs, HAs, and PSA are within the lead-like chemical space for all the ligands whilst the molecules have MW within the drug-like chemical space. The molecules are within the drug-like and known drug space (KDS) for Log P. The RBs are within the drug-like chemical space (for the definition of lead-like, drug-like and KDS regions see [54] and Table S3). Interestingly, it has been shown recently that orally bioavailable drugs tend not to follow the drug-like definition, in particular when it comes to MW [74]. The molecular weight of the ligands is between 303.5 and $335.6 \mathrm{~g} \mathrm{~mol}^{-1}$ and the Log P values lie in the range of 4.4 and 6.3. The ligands have low PSA values between 13.3 and 33.9. The known drug indexes $2 \mathrm{a}$ and $2 \mathrm{~b}$ $\left(\mathrm{KDIs}_{2 \mathrm{a} / 2 \mathrm{~b}}\right)$ [75] were also derived. The KDIs reflect the overall balance of the molecular properties 
of ligands based on KDS. $\mathrm{KDI}_{2 \mathrm{a}}$ is additive with a maximum of 6.0 and for $\mathrm{KDI}_{2 \mathrm{~b}}$ the indexes are multiplied giving 1.0 as its maximum. The average for $\mathrm{KDI}_{2 \mathrm{a}}$ for known drugs is $4.08( \pm 1.27)$ and the ligands lie in the range of 4.58 to 5.23 (i.e., they are well balanced compared to known drugs). The $\mathrm{KDI}_{2 \mathrm{~b}}$ gives a range of 0.18 to 0.43 with the average of know drugs being $0.18( \pm 0.20)$. The relatively low values can be attributed to the high Log Ps of 46a and 47a. The $\mathrm{KDI}_{2 b}$ index is less forgiving, since on one low number sinks the index significantly due to its multiplicative nature. Nevertheless, the ligands are relatively well balanced.

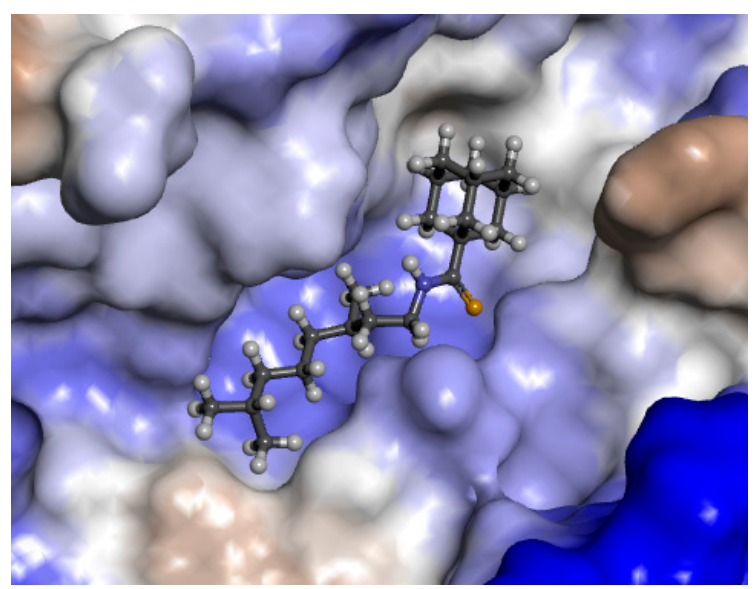

(A)

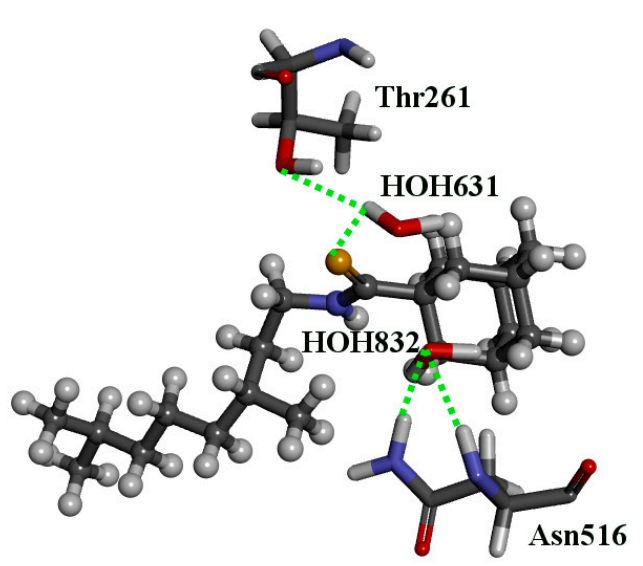

(B)

Figure 4. The docked configuration of $46 \mathrm{a}$ in the binding site of Tdp1 as predicted using the ChemScore scoring function. (A) The protein surface is rendered. The ligand occupies the binding pocket. Blue depicts a hydrophilic region on the surface; brown depicts hydrophobic region and whites shows neutral areas. (B) H-bonds are shown as dotted green lines between the sulphur atom and HOH631.

The water molecules also form H-bonds with Thr261 and Asn516.

\section{Conclusions}

Previously, a family of esters functionalised with monoterpene and adamantine moieties was found to be good inhibitors of Tdp1. Esters are known to be labile (i.e., hydrolyse relatively easily at physiological conditions, rendering compounds containing esters potentially problematic as drug candidates). We; therefore, substituted the ester bridge with amide and thioamide groups and tested them against Tdp1. Derivative 46a stood out as the most promising candidate as it synergised with topotecan, a Top1 poison in clinical use against cancer, only at $5 \mu \mathrm{M}$, which is a fully non-toxic dose. The reported $\mathrm{IC}_{50}$ value is $3.3 \mu \mathrm{M}$, which is not the lowest of the series synthesised, but interestingly on $46 \mathrm{a}$ showed synergy with topotecan in the cancer cell line used (A-549 lung adenocarcinoma. According to molecular modelling, a good fit is predicted for $46 \mathrm{a}$ into the substrate pocket of Tdp1, inhibiting its activity.

Supplementary Materials: The following are available online at http://www.mdpi.com/2076-3417/9/13/2767/s1, Table S1: Results of the scoring function for the ligands with and without two water molecules present; Table S2: The calculated molecular descriptors for the ligands; Table S3: Definition of lead-like, drug-like and known drug space (KDS) in terms of molecular descriptors. The values given are the maxima for each descriptor for the volumes of chemical space used; Figure S1: Intrinsic fluorescence spectroscopy of Tdp1 in the presence of compounds 45, 45a 46a, 47a and 51. Samples contained $10 \mu \mathrm{M}$ Tdp 1 and $1 \mathrm{mM}$ compound (if applicable) dissolved in a buffer containing $20 \mathrm{mM}$ Tris ( $\mathrm{pH} 8.0$ ) and $250 \mathrm{mM} \mathrm{NaCl}$. Control experiments were conducted with DMSO, which is the solvent that the compounds were dissolved in. Excitation wavelength was $280 \mathrm{~nm}$ and intrinsic fluorescence was measured between 300 and $450 \mathrm{~nm}$. Maximum fluorescence intensity was reported. Other compounds were not tested because they are not soluble at the required concentration in aqueous buffer; Figure S2: Protein melt curves showing the denaturing temperature of Tdp1 $(10 \mu \mathrm{M}$, red $)$ and $\mathrm{Tdp} 1(10 \mu \mathrm{M})$ in the presence of compound $44 \mathrm{~b}(100 \mu \mathrm{M}$, blue) as monitored by thermal shift assay. Buffer was $20 \mathrm{mM}$ Tris (pH 8.0) and $250 \mathrm{mM}$ $\mathrm{NaCl}$. Changes in denaturing temperature were found to be $-0.27^{\circ} \mathrm{C}$; Figure S3: Protein melt curves showing the denaturing temperature of Tdp1 $(10 \mu \mathrm{M}$, red) and Tdp1 $(10 \mu \mathrm{M})$ in the presence of compound $45 \mathrm{a}(100 \mu \mathrm{M}$, blue) as monitored by thermal shift assay. Buffer was $20 \mathrm{mM}$ Tris (pH 8.0) and $250 \mathrm{mM} \mathrm{NaCl}$. Changes in denaturing 
temperature were found to be $0.04{ }^{\circ} \mathrm{C}$; Figure S4: Protein melt curves showing the denaturing temperature of $\mathrm{Tdp} 1(10 \mu \mathrm{M}$, red) and Tdp1 $(10 \mu \mathrm{M})$ in the presence of compound $46 \mathrm{a}(100 \mu \mathrm{M}$, blue $)$ as monitored by thermal shift assay. Buffer was $20 \mathrm{mM}$ Tris ( $\mathrm{pH} 8.0$ ) and $250 \mathrm{mM} \mathrm{NaCl}$. Changes in denaturing temperature were found to be $-0.1^{\circ} \mathrm{C}$; Figure S5: Protein melt curves showing the denaturing temperature of Tdp1 $(10 \mu \mathrm{M}$, red $)$ and $\operatorname{Tdp} 1(10 \mu \mathrm{M})$ in the presence of compound $47 \mathrm{a}(100 \mu \mathrm{M}$, blue) as monitored by thermal shift assay. Buffer was $20 \mathrm{mM}$ Tris (pH 8.0) and $250 \mathrm{mM} \mathrm{NaCl}$. Changes in denaturing temperature were found to be $0.08{ }^{\circ} \mathrm{C}$; Figure S6: Protein melt curves showing the denaturing temperature of $\operatorname{Tdp} 1(10 \mu \mathrm{M}$, red $)$ and $\operatorname{Tdp} 1(10 \mu \mathrm{M})$ in the presence of compound $51(100 \mu \mathrm{M}$, blue) as monitored by thermal shift assay. Buffer was $20 \mathrm{mM}$ Tris (pH 8.0) and $250 \mathrm{mM}$ $\mathrm{NaCl}$. Changes in denaturing temperature were found to be $-0.22{ }^{\circ} \mathrm{C}$; Figures $\mathrm{S} 7-15:{ }^{1} \mathrm{H}$ NMR spectra of the products; Figure S16: Dose-dependent influence of the adamantane derivatives on A-549 cell viability using the MTT assay.

Author Contributions: Synthetic work, writing—original draft preparation, E.S.M., A.A.M., E.V.S. and K.P.V.; performing Tdp1 assay, cytotoxicity assay, A.A.C., A.L.Z. and O.D.Z.; compounds characterisation by NMR, D.V.K.; performing melting point and intrinsic tryptophan protein fluorescence experiments, J.P. and I.K.H.L.; molecular modelling and chemical space, D.M.A.-T. and J.R.; supervision, project administration, writing-review and editing, K.P.V., I.K.H.L., J.R., A.L.Z., N.F.S. and O.I.L.

Funding: This work was supported by the Russian Science Foundation (grant 19-13-00040).

Acknowledgments: Authors would like to acknowledge the Multi-Access Chemical Research Center SB RAS for spectral and analytical measurements. The cell line A-549 was obtained from the Russian Cell Culture Collection (RCCC) Institute of Cytology RAS, St. Petersburg, Russia.

Conflicts of Interest: The authors declare no conflict of interest.

\section{References}

1. Li, F.; Jiang, T.; Li, Q.; Ling, X. Camptothecin (CPT) and its derivatives are known to target topoisomerase I (Top1) as their mechanism of action: Did we miss something in CPT analogue molecular targets for treating human disease such as cancer? Am. J. Cancer Res. 2017, 7, 2350-2394. [PubMed]

2. Hevener, K.E.; Verstak, T.A.; Lutat, K.E.; Riggsbee, D.L.; Mooney, J.W. Recent developments in topoisomerase-targeted cancer chemotherapy. Acta Pharm. Sin. B 2018, 8, 844-861. [CrossRef]

3. Hsiang, Y.H.; Hertzberg, R.; Hecht, S.; Liu, L.F. Camptothecin induces protein-linked DNA breaks via mammalian DNA topoisomerase I. J. Biol. Chem. 1985, 260, 14873-14878. [PubMed]

4. Cuya, S.M.; Bjornsti, M.-A.; van Waardenburg, R.C.A.M. DNA topoisomerase-targeting chemotherapeutics: what's new? Cancer Chemother. Pharmacol. 2017, 80, 1-14. [CrossRef] [PubMed]

5. Pommier, Y. Topoisomerase I inhibitors: Camptothecins and beyond. Nat. Rev. Cancer 2006, 6, 789-802. [CrossRef] [PubMed]

6. Iyama, T.; Wilson, D.M. DNA repair mechanisms in dividing and non-dividing cells. DNA Repair 2013, 12, 620-636. [CrossRef] [PubMed]

7. Lebedeva, N.A.; Rechkunova, N.I.; Lavrik, O.I. AP-site cleavage activity of tyrosyl-DNA phosphodiesterase 1. FEBS Lett. 2011, 585, 683-686. [CrossRef]

8. Pommier, Y.; Redon, C.; Rao, V.A.; Seiler, J.A.; Sordet, O.; Takemura, H.; Antony, S.; Meng, L.H.; Liao, Z.Y.; Kohlhagen, G. Repair of and checkpoint response to topoisomerase I-mediated DNA damage. Mutat. Res. -Fundam. Mol. Mech. Mutagen. 2003, 532, 173-203. [CrossRef] [PubMed]

9. Jakobsen, A.-K.; Lauridsen, K.L.; Samuel, E.B.; Proszek, J.; Knudsen, B.R.; Hager, H.; Stougaard, M. Correlation between topoisomerase I and tyrosyl-DNA phosphodiesterase 1 activities in non-small cell lung cancer tissue. Exp. Mol. Pathol. 2015, 99, 56-64. [CrossRef]

10. Meisenberg, C.; Gilbert, D.C.; Chalmers, A.; Haley, V.; Gollins, S.; Ward, S.E.; El-Khamisy, S.F. Clinical and Cellular Roles for TDP1 and TOP1 in Modulating Colorectal Cancer Response to Irinotecan. Mol. Cancer Ther. 2015, 14, 575-585. [CrossRef]

11. Perego, P.; Cossa, G.; Tinelli, S.; Corna, E.; Carenini, N.; Gatti, L.; De Cesare, M.; Ciusani, E.; Zunino, F.; Luison, E. Role of tyrosyl-DNA phosphodiesterase 1 and inter-players in regulation of tumor cell sensitivity to topoisomerase I inhibition. Biochem. Pharmacol. 2012, 83, 27-36. [CrossRef] [PubMed]

12. Murai, J.; Huang, S.N.; Das, B.B.; Dexheimer, T.S.; Takeda, S.; Pommier, Y. Tyrosyl-DNA Phosphodiesterase 1 (TDP1) Repairs DNA Damage Induced by Topoisomerases I and II and Base Alkylation in Vertebrate Cells. J. Biol. Chem. 2012, 287, 12848-12857. [CrossRef] 
13. Alagoz, M.; C. Gilbert, D.; El-Khamisy, S.; J. Chalmers, A. DNA Repair and Resistance to Topoisomerase I Inhibitors: Mechanisms, Biomarkers and Therapeutic Targets. Curr. Med. Chem. 2012, 19, 3874-3885. [CrossRef] [PubMed]

14. Pommier, Y.; Huang, S.N.; Gao, R.; Das, B.B.; Murai, J.; Marchand, C. Tyrosyl-DNA-phosphodiesterases (TDP1 and TDP2). DNA Repair 2014, 19, 114-129. [CrossRef] [PubMed]

15. Blasiak, J. DNA-Damaging Anticancer Drugs-A Perspective for DNA Repair-Oriented Therapy. Curr. Med. Chem. 2017, 24, 1488-1503. [CrossRef] [PubMed]

16. Zakharenko, A.L.; Luzina, O.A.; Sokolov, D.N.; Zakharova, O.D.; Rakhmanova, M.E.; Chepanova, A.A.; Dyrkheeva, N.S.; Lavrik, O.I.; Salakhutdinov, N.F. Usnic acid derivatives are effective inhibitors of tyrosyl-DNA phosphodiesterase 1. Russ. J. Biorgan. Chem. 2017, 43, 84-90. [CrossRef]

17. Liao, Z.; Thibaut, L.; Jobson, A.; Pommier, Y. Inhibition of human Tyrosyl-DNA Phosphodiesterase (Tdp1) by aminoglycoside antibiotics and ribosome inhibitors. Mol. Pharmacol. 2006, 70, 366-372. [CrossRef]

18. Komarova, A.O.; Drenichev, M.S.; Dyrkheeva, N.S.; Kulikova, I.V.; Oslovsky, V.E.; Zakharova, O.D.; Zakharenko, A.L.; Mikhailov, S.N.; Lavrik, O.I. Novel group of tyrosyl-DNA-phosphodiesterase 1 inhibitors based on disaccharide nucleosides as drug prototypes for anti-cancer therapy. J. Enzyme Inhib. Med. Chem. 2018, 33, 1415-1429. [CrossRef]

19. Nguyen, T.X.; Morrell, A.; Conda-Sheridan, M.; Marchand, C.; Agama, K.; Bermingam, A.; Stephen, A.G.; Chergui, A.; Naumova, A.; Fisher, R. Synthesis and Biological Evaluation of the First Dual Tyrosyl-DNA Phosphodiesterase I (Tdp1)-Topoisomerase I (Top1) Inhibitors. J. Med. Chem. 2012, 55, 4457-4478. [CrossRef]

20. Cushman, M.S.; Wang, P.; Pomier, Y.G.; Elsayed, M.S.A. Azaindenoisoquinoline Compounds and Uses Thereof. Patent Application WO 2018/118852 A1, 28 June 2018.

21. Cushman, M.S.; Beck, D.E.; Pomier, Y.G. Aza-A-Ring Indenoisoquinoline Topoisomerase I Poisons. Patent Application WO2017160898A1, 21 September 2017.

22. Beck, D.E.; Reddy, P.V.N.; Lv, W.; Abdelmalak, M.; Tender, G.S.; Lopez, S.; Agama, K.; Marchand, C.; Pommier, Y.; Cushman, M. Investigation of the Structure-Activity Relationships of Aza-A-Ring Indenoisoquinoline Topoisomerase I Poisons. J. Med. Chem. 2016, 59, 3840-3853. [CrossRef]

23. Pommier, Y.; Thibaut, L.; Marchand, C. Tetracycline Compounds and Methods of Treatment. Patent Application WO 2007/112121 A2, 26 June 2001.

24. Zhang, X.-R.; Wang, H.-W.; Tang, W.-L.; Zhang, Y.; Yang, H.; Hu, D.-X.; Ravji, A.; Marchand, C.; Kiselev, E.; Ofori-Atta, K. Discovery, Synthesis, and Evaluation of Oxynitidine Derivatives as Dual Inhibitors of DNA Topoisomerase IB (TOP1) and Tyrosyl-DNA Phosphodiesterase 1 (TDP1), and Potential Antitumor Agents. J. Med. Chem. 2018, 61, 9908-9930. [CrossRef] [PubMed]

25. Dexheimer, T.S.; Gediya, L.K.; Stephen, A.G.; Weidlich, I.; Antony, S.; Marchand, C.; Interthal, H.; Nicklaus, M.; Fisher, R.J.; Njar, V.C. 4-Pregnen-21-ol-3,20-dione-21-(4-bromobenzenesufonate) (NSC 88915) and related novel steroid derivatives as tyrosyl-DNA phosphodiesterase (Tdp1) inhibitors. J. Med. Chem. 2009, 52, 7122-7131. [CrossRef] [PubMed]

26. Pommier, Y.; Marchand, C.; Wang, Z.; Sirivolu, V.R. Thioxothiazolidinone Derivatives Useful as Inhibitors of Tdp1. WO2013/055771 A1, 18 April 2013.

27. Sirivolu, V.R.; Vernekar, S.K.V.; Marchand, C.; Naumova, A.; Chergui, A.; Renaud, A.; Stephen, A.G.; Chen, F.; Sham, Y.Y.; Pommier, Y. 5-Arylidenethioxothiazolidinones as Inhibitors of Tyrosyl-DNA Phosphodiesterase I. J. Med. Chem. 2012, 55, 8671-8684. [CrossRef] [PubMed]

28. Jun, J.H.; Kumar, V.; Dexheimer, T.S.; Wedlich, I.; Nicklaus, M.C.; Pommier, Y.; Malhotra, S.V. Synthesis, anti-cancer screening and tyrosyl-DNA phosphodiesterase 1 (Tdp1) inhibition activity of novel piperidinyl sulfamides. Eur. J. Pharm. Sci. 2018, 111, 337-348. [CrossRef] [PubMed]

29. Zakharenko, A.; Khomenko, T.; Zhukova, S.; Koval, O.; Zakharova, O.; Anarbaev, R.; Lebedeva, N.; Korchagina, D.; Komarova, N.; Vasiliev, V. Synthesis and biological evaluation of novel tyrosyl-DNA phosphodiesterase 1 inhibitors with a benzopentathiepine moiety. Biorgan. Med. Chem. 2015, 23, 2044-2052. [CrossRef] [PubMed]

30. Pommier, Y.; Marchand, C.; Selvam, P.; Dexheimer, T.; Maddali, K. Fluoroquinolone Derivatives or Sulfonamide Moiety-Containing Compounds as Inhibitors of Tyrosyl-Dnaphosphodiesterase (TDP1). US8716295 (B2), 6 May 2014.

31. Pommier, Y.; Marchand, C. Diamidine Inhibitors of TDP1. WO2007/126857 A1, 8 November 2007. 
32. Antony, S.; Marchand, C.; Stephen, A.G.; Thibaut, L.; Agama, K.K.; Fisher, R.J.; Pommier, Y. Novel high-throughput electrochemiluminescent assay for identification of human tyrosyl-DNA phosphodiesterase (Tdp1) inhibitors and characterization of furamidine (NSC 305831) as an inhibitor of Tdp1. Nucleic Acids Res. 2007, 35, 4474-4484. [CrossRef] [PubMed]

33. Zakharova, O.; Luzina, O.; Zakharenko, A.; Sokolov, D.; Filimonov, A.; Dyrkheeva, N.; Chepanova, A.; Ilina, E.; Ilyina, A.; Klabenkova, K. Synthesis and evaluation of aryliden- and hetarylidenfuranone derivatives of usnic acid as highly potent Tdp1 inhibitors. Biorgan. Med. Chem. 2018, 26, 4470-4480. [CrossRef]

34. Dyrkheeva, N.; Luzina, O.; Filimonov, A.; Zakharova, O.; Ilina, E.; Zakharenko, A.; Kuprushkin, M.; Nilov, D.; Gushchina, I.; Švedas, V. Inhibitory Effect of New Semisynthetic Usnic Acid Derivatives on Human Tyrosyl-DNA Phosphodiesterase 1. Planta Med. 2019, 85, 103-111. [CrossRef]

35. Zakharenko, A.L.; Luzina, O.A.; Sokolov, D.N.; Kaledin, V.I.; Nikolin, V.P.; Popova, N.A.; Patel, J.; Zakharova, O.D.; Chepanova, A.A.; Zafar, A. Novel tyrosyl-DNA phosphodiesterase 1 inhibitors enhance the therapeutic impact of topotecan on in vivo tumor models. Eur. J. Med. Chem. 2019, 161, 581-593. [CrossRef]

36. Zakharenko, A.; Luzina, O.; Koval, O.; Nilov, D.; Gushchina, I.; Dyrkheeva, N.; Švedas, V.; Salakhutdinov, N.; Lavrik, O. Tyrosyl-DNA Phosphodiesterase 1 Inhibitors: Usnic Acid Enamines Enhance the Cytotoxic Effect of Camptothecin. J. Nat. Prod. 2016, 79, 2961-2967. [CrossRef]

37. Bermingham, A.; Price, E.; Marchand, C.; Chergui, A.; Naumova, A.; Whitson, E.L.; Krumpe, L.R.H.; Goncharova, E.I.; Evans, J.R.; McKee, T.C. Identification of Natural Products That Inhibit the Catalytic Function of Human Tyrosyl-DNA Phosphodiesterase (TDP1). SLAS Discov. Adv. Life Sci. R D 2017, 22, 1093-1105. [CrossRef]

38. Tian, L.-W.; Feng, Y.; Tran, T.D.; Shimizu, Y.; Pfeifer, T.; Vu, H.T.; Quinn, R.J. Achyrodimer F, a tyrosyl-DNA phosphodiesterase I inhibitor from an Australian fungus of the family Cortinariaceae. Biorgan. Med. Chem. Lett. 2017, 27, 4007-4010. [CrossRef] [PubMed]

39. Tian, L.-W.; Feng, Y.; Tran, T.D.; Shimizu, Y.; Pfeifer, T.; Forster, P.I.; Quinn, R.J. Tyrosyl-DNA Phosphodiesterase I Inhibitors from the Australian Plant Macropteranthes leichhardtii. J. Nat. Prod. 2015, 78, 1756-1760. [CrossRef] [PubMed]

40. Weidlich, I.E.; Dexheimer, T.; Marchand, C.; Antony, S.; Pommier, Y.; Nicklaus, M.C. Inhibitors of human tyrosyl-DNA phospodiesterase (hTdp1) developed by virtual screening using ligand-based pharmacophores. Biorgan. Med. Chem. 2010, 18, 182-189. [CrossRef]

41. Salakhutdinov, N.F.; Volcho, K.P.; Yarovaya, O.I. Monoterpenes as a renewable source of biologically active compounds. Pure Appl. Chem. 2017, 89, 1105-1117. [CrossRef]

42. Khomenko, T.; Zakharenko, A.; Odarchenko, T.; Arabshahi, H.J.; Sannikova, V.; Zakharova, O.; Korchagina, D.; Reynisson, J.; Volcho, K.; Salakhutdinov, N. New inhibitors of tyrosyl-DNA phosphodiesterase I (Tdp 1) combining 7-hydroxycoumarin and monoterpenoid moieties. Biorgan. Med. Chem. 2016, 24, 5573-5581. [CrossRef]

43. Mozhaitsev, E.; Suslov, E.; Demidova, Y.; Korchagina, D.; Volcho, K.; Zakharenko, A.; Vasil'eva, I.; Kupryushkin, M.; Chepanova, A.; Ayine-Tora, D.M. The Development of Tyrosyl-DNA Phosphodyesterase 1 (TDP1) Inhibitors Based on the Amines Combining Aromatic/Heteroaromatic and Monoterpenoid Moieties. Lett. Drug Des. Discov. 2019, 16, 597-605. [CrossRef]

44. Salomatina, O.; Popadyuk, I.; Zakharenko, A.; Zakharova, O.; Fadeev, D.; Komarova, N.; Reynisson, J.; Arabshahi, H.; Chand, R.; Volcho, K. Novel Semisynthetic Derivatives of Bile Acids as Effective Tyrosyl-DNA Phosphodiesterase 1 Inhibitors. Molecules 2018, 23, 679. [CrossRef]

45. Li-Zhulanov, N.; Zakharenko, A.; Chepanova, A.; Patel, J.; Zafar, A.; Volcho, K.; Salakhutdinov, N.; Reynisson, J.; Leung, I.; Lavrik, O. A Novel Class of Tyrosyl-DNA Phosphodiesterase 1 Inhibitors That Contains the Octahydro-2H-chromen-4-ol Scaffold. Molecules 2018, 23, 2468. [CrossRef]

46. Zakharenko, A.L.; Ponomarev, K.U.; Suslov, E.V.; Korchagina, D.V.; Volcho, K.P.; Vasil'eva, I.A.; Salakhutdinov, N.F.; Lavrik, O.I. Inhibitory properties of nitrogen-containing adamantane derivatives with monoterpenoid fragments against tyrosyl-DNA phosphodiesterase 1. Russ. J. Biorgan. Chem. 2015, 41, 657-662. [CrossRef]

47. Ponomarev, K.Y.; Suslov, E.V.; Zakharenko, A.L.; Zakharova, O.D.; Rogachev, A.D.; Korchagina, D.V.; Zafar, A.; Reynisson, J.; Nefedov, A.A.; Volcho, K.P. Aminoadamantanes containing monoterpene-derived fragments as potent tyrosyl-DNA phosphodiesterase 1 inhibitors. Biorgan. Chem. 2018, 76, 392-399. [CrossRef] [PubMed] 
48. Zakharenko, A.L.; Mozhaitsev, E.S.; Suslov, E.V.; Korchagina, D.V.; Volcho, K.P.; Salakhutdinov, N.F.; Lavrik, O.I. Synthesis and Inhibitory Properties of Imines Containing Monoterpenoid and Adamantane Fragments Against DNA Repair Enzyme Tyrosyl-DNA Phosphodiesterase 1 (Tdp1). Chem. Nat. Compd. 2018, 54, 672-676. [CrossRef]

49. Mozhaitsev, E.S.; Zakharenko, A.L.; Suslov, E.V.; Korchagina, D.V.; Zakharova, O.D.; Vasil'eva, I.A.; Chepanova, A.A.; Black, E.; Patel, J.; Chand, R. Novel Inhibitors of DNA Repair Enzyme TDP1 Combining Monoterpenoid and Adamantane Fragments. Anticancer Agents Med. Chem. 2018, 19, 463-472. [CrossRef] [PubMed]

50. Laizure, S.C.; Herring, V.; Hu, Z.; Witbrodt, K.; Parker, R.B. The role of human carboxylesterases in drug metabolism: Have we overlooked their importance? Pharmacotherapy 2013, 33, 210-222. [CrossRef] [PubMed]

51. Akgun, B.; Hall, D.G. Fast and Tight Boronate Formation for Click Bioorthogonal Conjugation. Angew. Chem. Int. Ed. 2016, 55, 3909-3913. [CrossRef] [PubMed]

52. Fukuyama, T.; Reding, M.T.; Kaburagi, Y.; Tokuyama, H. Synthesis of 2,3-Disubstituted Indoles by Radical Cyclization with Hypophosphorous Acid and Its Application to Total Synthesis of $( \pm)$-Catharanthine. Heterocycles 2002, 56, 313. [CrossRef]

53. Davies, D.R.; Interthal, H.; Champoux, J.J.; Hol, W.G.J. Insights into Substrate Binding and Catalytic Mechanism of Human Tyrosyl-DNA Phosphodiesterase (Tdp1) from Vanadate and Tungstate-inhibited Structures. J. Mol. Biol. 2002, 324, 917-932. [CrossRef]

54. Zhu, F.; Logan, G.; Reynisson, J. Wine Compounds as a Source for HTS Screening Collections. A Feasibility Study. Mol. Inform. 2012, 31, 847-855. [CrossRef]

55. Berman, H.; Henrick, K.; Nakamura, H. Announcing the worldwide Protein Data Bank. Nat. Struct. Mol. Biol. 2003, 10, 980. [CrossRef]

56. Scigress, version FJ 2.6 (EU 3.1.7); Fujitsu Limited: Tokyo, Japan, 2008-2016.

57. Allinger, N.L. Conformational analysis. 130. MM2. A hydrocarbon force field utilizing V1 and V2 torsional terms. J. Am. Chem. Soc. 1977, 99, 8127-8134. [CrossRef]

58. Jones, G.; Willett, P.; Glen, R.C.; Leach, A.R.; Taylor, R. Development and validation of a genetic algorithm for flexible docking. J. Mol. Biol. 1997, 267, 727-748. [CrossRef] [PubMed]

59. Eldridge, M.D.; Murray, C.W.; Auton, T.R.; Paolini, G.V.; Mee, R.P. Empirical scoring functions: I. The development of a fast empirical scoring function to estimate the binding affinity of ligands in receptor complexes. J. Comput. Aided. Mol. Des. 1997, 11, 425-445. [CrossRef] [PubMed]

60. Verdonk, M.L.; Cole, J.C.; Hartshorn, M.J.; Murray, C.W.; Taylor, R.D. Improved protein-ligand docking using GOLD. Proteins Struct. Funct. Bioinform. 2003, 52, 609-623. [CrossRef] [PubMed]

61. Korb, O.; Stutzle, T.; Exner, T.E. Empirical Scoring Functions for Advanced Protein-Ligand Docking with PLANTS. J. Chem. Inf. Model. 2009, 49, 84-96. [CrossRef] [PubMed]

62. Mooij, W.T.M.; Verdonk, M.L. General and targeted statistical potentials for protein-ligand interactions. Proteins Struct. Funct. Bioinform. 2005, 61, 272-287. [CrossRef] [PubMed]

63. QikProp, version 3.2; Schrödinger: New York, NY, USA, 2009.

64. Ioakimidis, L.; Thoukydidis, L.; Mirza, A.; Naeem, S.; Reynisson, J. Benchmarking the Reliability of QikProp. Correlation between Experimental and Predicted Values. QSAR Comb. Sci. 2008, 27, 445-456. [CrossRef]

65. Interthal, H.; Pouliot, J.J.; Champoux, J.J. The tyrosyl-DNA phosphodiesterase Tdp1 is a member of the phospholipase D superfamily. Proc. Natl. Acad. Sci. USA. 2001, 98, 12009-12014. [CrossRef] [PubMed]

66. Mosmann, T. Rapid colorimetric assay for cellular growth and survival: Application to proliferation and cytotoxicity assays. J. Immunol. Methods 1983, 65, 55-63. [CrossRef]

67. Huang, R.; Ayine-Tora, D.M.; Muhammad Rosdi, M.N.; Li, Y.; Reynisson, J.; Leung, I.K.H. Virtual screening and biophysical studies lead to HSP90 inhibitors. Biorgan. Med. Chem. Lett. 2017, 27, 277-281. [CrossRef]

68. Molle, G.; Bauer, P.; Dubois, J.E. Formation of cage-structure organomagnesium compounds. Influence of the degree of adsorption of the transient species at the metal surface. J. Org. Chem. 1982, 47, 4120-4128. [CrossRef]

69. Madder, A.; Sebastian, S.; Van Haver, D.; De Clercq, P.J.; Maskill, H. Mechanism of esterification of 1,3-dimethylamino alcohols by $\mathrm{N}$-acetylimidazole in acetonitrile and the influence of alkyl and geminal dialkyl substitution upon the rate. J. Chem. Soc. Perkin Trans. 1997, 2, 2787-2793. [CrossRef]

70. Mukherjee, A.; Wu, Q.; le Noble, W.J. Face Selection in Claisen Rearrangements. J. Org. Chem. 1994, 59, 3270-3274. [CrossRef] 
71. Pantoliano, M.W.; Petrella, E.C.; Kwasnoski, J.D.; Lobanov, V.S.; Myslik, J.; Graf, E.; Carver, T.; Asel, E.; Springer, B.A.; Lane, P. High-Density Miniaturized Thermal Shift Assays as a General Strategy for Drug Discovery. J. Biomol. Screen. 2001, 6, 429-440. [CrossRef] [PubMed]

72. Jensen, P.W.; Falconi, M.; Kristoffersen, E.L.; Simonsen, A.T.; Cifuentes, J.B.; Marcussen, L.B.; Frøhlich, R.; Vagner, J.; Harmsen, C.; Juul, S. Real-time detection of TDP1 activity using a fluorophore-quencher coupled DNA-biosensor. Biosens. Bioelectron. 2013, 48, 230-237. [CrossRef] [PubMed]

73. Martino, E.; Della Volpe, S.; Terribile, E.; Benetti, E.; Sakaj, M.; Centamore, A.; Sala, A.; Collina, S. The long story of camptothecin: From traditional medicine to drugs. Biorgan. Med. Chem. Lett. 2017, 27, 701-707. [CrossRef] [PubMed]

74. Shultz, M.D. Two Decades under the Influence of the Rule of Five and the Changing Properties of Approved Oral Drugs. J. Med. Chem. 2019, 62, 1701-1714. [CrossRef] [PubMed]

75. Eurtivong, C.; Reynisson, J. The Development of a Weighted Index to Optimise Compound Libraries for High Throughput Screening. Mol. Inform. 2019, 38, 1800068-1800078. [CrossRef]

(C) 2019 by the authors. Licensee MDPI, Basel, Switzerland. This article is an open access article distributed under the terms and conditions of the Creative Commons Attribution (CC BY) license (http://creativecommons.org/licenses/by/4.0/). 